\title{
Meta-Analysis of the Reversible Inhibitors of Monoamine Oxidase Type A Moclobemide and Brofaromine for the Treatment of Depression
}

\author{
Francisco Lotufo-Neto, M.D., Ph.D., Madhukar Trivedi, M.D., and Michael E. Thase, M.D.
}

The reversible inhibitors of monoamine oxidase type $A$ (RIMAs) are a newer group of antidepressants that have had much less impact on clinical psychopharmacology than another contemporary class of medications, the selective serotonin reuptake-inhibitors (SSRIs). The RIMAs agents are distinguished from the older monoamine oxidase inhibitors (MAOIs) by their selectivity and reversibility. As a result, dietary restrictions are not required during RIMA therapy, and hypertensive crises are quite rare. In this article, we describe a series of meta-analyses of studies of the two most widely researched RIMAs, moclobemide (MOC; Aurorex) and brofaromine (BRO). Our findings confirm that both $B R O$ and $M O C$ are as effective as the tricyclic antidepressants, and they are better tolerated. However, $B R O$ is not being studied at present for reasons unrelated to efficacy or side effects. MOC, which is available throughout much of the world (but not the United States), is significantly more effective than placebo and, at the least, comparable to the SSRIs in both efficacy and tolerability. For MOC, higher dosages may enhance efficacy for more severe depressions. We also found evidence that supports clinical impressions that MOC is somewhat less effective, albeit better tolerated, than older MAOIs, such as phenelzine or tranylcypromine. Little evidence has yet emerged to suggest that the RIMAs share older MAOIs' utility for treatment of depressions characterized by prominent reverse neurovegetative features. Based on available evidence, the RIMAs appear to have a limited, but useful, role in the differential therapeutics of the depressive disorders. [Neuropsychopharmacology 20:226-247, 1999] (C) 1999 American College of Neuropsychopharmacology. Published by Elsevier Science Inc.
KEY WORDS: RIMAs; MAOIs; Brofaromine; Moclobemide; Antidepressants

The monoamine oxidase inhibitors (MAOIs) are an important class of antidepressants that have been in use for nearly 40 years, often with controversy because of

From the Instituto de Psiquiatria da Faculdade de Medicina da Universidade de São Paulo (FL-N), São Paulo, Brazil; University of Texas (MT), Southwestern Medical Center, Dallas, Texas; and University of Pittsburgh School of Medicine (MET), Western Psychiatric Institute and Clinic, Pittsburgh, Pennsylvania.

Address correspondence to: Michael Thase, University of Pittsburgh School of Medicine, Western Psychiatric Institute and Clinic, 3811 O'Hara Street, Pittsburgh, PA 15213.

Received January 28, 1998; revised July 8, 1998; accepted July 22, 1998. concerns about both efficacy and safety (Laux et al. 1995; Thase et al. 1995). Among a number of MAOIs introduced in the 1950s and 1960s, only two remain available in the United States, Phenelzine (Nardil) and tranylcypromine (Parnate). For several reasons, these MAOIs are now usually relegated to the role of third- or even fourth-line treatments (Thase and Rush 1995). For example, significant cardiovascular side effects-both orthostatic hypotension and the sporadic incidence of hypertensive crises (the so-called "cheese reaction") occasionally necessitate the discontinuation of an otherwise beneficial treatment. In the latter case, adherence to a low tyramine diet is difficult for some and, at the least, inconvenient for most. Nevertheless, the efficacy of the MAOIs, particularly for patients with treatment- 
resistant, bipolar, and atypical depressive syndromes, has sustained interest in this class of medication (Nutt and Glue, 1989; Quitkin et al. 1993; Thase et al. 1995). For the past 20 years, considerable effort has been expended to develop safer and better tolerated MAOIs. A subclass of MAOIs, reversible inhibitors of monoamine oxidase type A (RIMAs), has, arguably, accomplished this goal. This article briefly reviews the clinical pharmacology of the RIMAs and presents meta-analyses of the efficacy of the two most widely studied compounds, moclobemide (Aurorex) and brofaromine.

\section{PHARMACOLOGY}

Many of the problems associated with phenelzine and tranylcypromine treatment result from two shared pharmacologic characteristics: irreversibility and nonselectivity. Irreversibility refers to the tenacious binding of the drug to the MAO enzyme, essentially for the "lifetime" of the molecule (i.e., 14-28 days). Thus, even a high concentration of substrate cannot displace an irreversible MAOI from the enzyme. This is why normally innocuous concentrations of substrates with vasopressor effects, such as tyramine or pseudoephedrine, can be lethal if ingested during treatment with an irreversible MAOI.

Nonselectivity refers to the tendency for phenelzine and tranylcypromine to bind to both the $\mathrm{A}$ and $\mathrm{B}$ isozymes of MAO (Johnston, 1968). MAO-A is found in the brain, small intestine, liver, portal system, and peripheral adrenergic neurons (Youdim et al. 1988), and it is relatively selective for metabolism of norepinephrine and serotonin. MAO-B, which is found in blood platelets, the brain and other tissues, is relatively selective for metabolism of benzylamine and phenylethylamine (Youdim et al. 1988). Tyramine and dopamine are metabolized by both MAO-A and MAO-B.

It has been established that hypertensive crises are a consequence of MAO-A inhibition (Youdim et al. 1988; Laux et al. 1995). In vivo inhibition of MAO with either irreversible or nonselective compounds permits the uptake of high concentrations of tyramine and other sympathomimetics into the blood circulation, where they gain access to peripheral adrenergic neurons, trigger catecholamine release, and cause a marked and rapid increase in blood pressure (Lavian et al. 1993).

One of the first selective MAOIs to be identified was clorgyline (Lipper et al. 1979). Clorgyline is a potent inhibitor of MAO-A that has significant antidepressant effects (Lipper et al. 1979; Potter et al. 1982). However, clorgyline is an irreversible enzyme inhibitor, and has some liability to cause hypertensive crises in the face of high substrate concentrations, despite its selectivity (Laux et al. 1995). Thus, clorgyline was not considered to represent much of an advance over the nonselective MAOIs and was not vigorously pursued.
The ready accessibility of the blood platelet for clinical studies helped to facilitate identification of agents that were selective for the MAO-B isozyme. The potential therapeutic promise of B-selective MAOIs was further heightened by evidence linking percentage inhibition of platelet (type B) MAO with response to phenelzine (e.g., Robinson et al. 1978). In 1972, Knoll and Magyar first described L-selegiline (Eldepryl), a compound structurally similar to clorgyline but, instead, a selective inhibitor of MAO type B. Although L-selegiline is also an irreversible MAOI, it is relatively selective for inhibition of the MAO-B isozyme at doses $<20 \mathrm{mg} /$ day, and it is unlikely to potentiate the sympathomimetic effects of tyramine (Laux et al. 1995).

Clinical trials did not establish L-selegiline as a potent antidepressant at doses selective for MAO Type B inhibition (i.e., $5-15 \mathrm{mg} /$ day). In fact, the best evidence of antidepressant efficacy comes from studies employing larger, nonselective dosages (i.e., $20-50 \mathrm{mg}$ /day) of L-selegiline (e.g., Mann et al. 1989; Sunderland et al. 1994). This suggests that inhibition of type A enzyme, either alone or in combination with inhibition of MAO Type B, is critical to the MAOIs' antidepressant response (Thase et al. 1995). L-selegiline is, however, a useful treatment of Parkinson's disease in doses that are selective for Type B inhibition.

\section{Moclobemide}

Moclobemide (p-chloro-N-[2-morpholinoethyl] benzamide was synthesized in 1972 by P.-C. Wyss in Basel, Switzerland. The compound was initially developed as an antihyperlipidemic, but when it did not show the expected therapeutic activity, it was submitted for pharmacologic testing to determine central nervous system activity. Shortly thereafter, the MAO inhibitory action of moclobemide (MOC) was identified (see Haefely et al. 1993). MOC was the first reversible MAO-A inhibitor to be approved for general use in the United Kingdom and Europe (Youdim et al. 1988; Haefely et al. 1992; Haefely et al. 1993); it is now available in more than 50 countries worldwide (Chen and Ruch, 1993). MOC is prescribed less frequently than the SSRIs in most countries in which both classes of medication are available. However, there are notable exceptions, and MOC is widely used as a first-line antidepressant in Finland and Australia.

MOC treatment is seldom associated with hypertensive crises, even when tyramine ingestion exceeds the concentrations that are present in cheese, red wine, or beer (Laux et al. 1995). MOC is less likely to cause a hypertensive crisis than clorgyline because it is readily displaced by tyramine from its binding site on MAO-A (Youdim, 1995). Thus, it takes about 8 times more tyramine (i.e., $63 \mathrm{mg}$ ) to elicit a $30-\mathrm{mm} / \mathrm{Hg}$ rise in supine diastolic blood pressure during MOC treatment as compared to tranylcypromine treatment (i.e., $8 \mathrm{mg}$ ty- 
ramine) (Laux et al. 1995). Further, because tyramine does not normally cross the blood-brain barrier, central MAO-A will remain inhibited irrespective of dietary intake. Reversibility thus should not alter the RIMAs effects on norepinephrine and serotonin in the central nervous system (Korn et al. 1988).

MOC has linear pharmacokinetics and a wide therapeutic index. Amounts as high as 20,000 $\mathrm{mg}$ have been ingested without fatality (Laux et al. 1995). After oral MOC administration, $95 \%$ of the dose is recovered in the urine within 4 days, with a mean of $92 \%$ excreted during the first $12 \mathrm{~h}$. Although highly protein bound, the elimination half-life of MOC ranges from 1 to $3 \mathrm{~h}$, and metabolism is not greatly affected by age or diet (Laux et al. 1995).

MOC is extensively metabolized by the liver; 19 metabolites have been identified, accounting for $64 \%$ of the oral dose (Jauch et al. 1990). The metabolite pattern in the plasma has been found to be qualitatively, but not quantitatively, similar to the pattern observed in the urine, with all of the main urinary metabolites also found in the plasma (Jauch et al. 1990).

MOC is typically prescribed on a twice or three times per day schedule. The therapeutic dosage usually ranges between 300 and $450 \mathrm{mg} /$ day, although some patients benefit from dosages of $900 \mathrm{mg} /$ day or even higher. Common side effects include nausea, insomnia, tremor, and lightheadedness. Orthostatic hypotension is uncommon, however, even among the elderly. Single daily dosing has been evaluated (see Paykel 1995) and appears to be effective, although it may be associated with an increase in nuisance side effects.

\section{Brofaromine}

Brofaromine [4-(5-methoxy-7-bromo-benzofuranyl)-2piperidine HCL] was first synthesized in the early 1980s, and its potential as an antidepressant was rapidly appreciated (e.g., Waldmeier et al. 1993). The main pharmacodynamic difference between BRO and MOC is that the former compound also is a modest inhibitor of serotonin reuptake, with about $20 \%$ of the potency of fluoxetine (Prozac) (Waldmeier et al. 1993). This additional pharmacodynamic effect could, theoretically, enhance therapeutic potency, although it could also convey an increased risk of serotoninergic toxicity, particularly following overdose. Specifically, a number of fatalities were observed when older MAOIs were used shortly after discontinuation of fluoxetine (Beasley et al. 1993). There are, however, insufficient data concerning either this potential beneficial effect or its risk, and the reversibility of MAO inhibition provides some degree of protection against development of serotonin syndrome (see, for example, Joffe and Bakish 1994).

Similarities between BRO and MOC include short duration of MAO-A inhibition and reversible displace- ment from the enzymatic site by substrates such as tyramine, dopamine, and serotonin.

BRO has a half-life of 9 to $14 \mathrm{hrs}$ (Waldmeier et al. 1993). It is absorbed from the gut at a slower rate than MOC, reaching peak plasma concentrations within 2 to $4 \mathrm{~h}$ after oral ingestion (Waldmeier et al. 1993). Brofaromine is extensively $(98 \%)$ protein bound and, when compared to MOC, it is more tightly bound to MAO-A (Volz et al. 1995). For example, it takes $44 \mathrm{mg}$ of tyramine to cause a $30-\mathrm{mmHg}$ increase in supine diastolic blood pressure during BRO treatment (Laux et al. 1995). In relative terms, this is a fivefold increase in tolerability to the pressor effects of tyramine as compared to tranylcypromine. However, it is only about twothirds the amount of tyramine required to produce the same effect as observed during treatment with MOC (Laux et al. 1995). BRO is metabolized by the liver and has no active metabolites (Waldmeier et al. 1993). Approximately 8 days are required to "wash out" the effects of BRO as measured by blood pressure effects during the oral tyramine challenge test (Laux et al. 1995).

Brofaromine may be prescribed once or twice a day. Available evidence suggests optimal therapeutic activity is observed at doses between 50 and $200 \mathrm{mg} /$ day (Laux et al. 1995; Volz et al. 1994b). Higher doses have not been studied extensively, and there is not a wealth of "open label" clinical experience with this compound. As with MOC, dose-limiting side effects typically include nausea, insomnia, and tremor.

Brofaromine is currently not being investigated anywhere in the world. This is an apparent consequence of a corporate decision that it would not be cost-effective to conduct the additional placebo-controlled studies that would be necessary to receive regulatory approval (Volz et al. 1995). It is assumed that this decision was influenced by the relatively small market share of MOC (vis à vis the SSRIs) in most western countries.

Other RIMAs in development include amiflamine, befloxatone, cimoxatone, and toloxatone (see Laux et al. 1995). However, none of these compounds have yet been studied extensively and, thus, they will not be included in this review.

We next use meta-analysis to quantify the absolute and relative efficacies of MOC and BRO. Efficacy is determined with respect to placebo (PBO) control groups and standard tricyclic antidepressant (TCA), SSRI, and MAOI comparators, as well as (when available) other classes of antidepressants.

\section{METHODS}

\section{Literature Review}

We reviewed all relevant literature published in English language journals before March, 1996. An initial literature review was conducted using MEDLINE and 
Psychological Abstracts, targeting the following key words: monoamine oxidase inhibitors, RIMAs, moclobemide, and brofaromine. The reference lists of each of these articles also were reviewed to ensure that the literature review was comprehensive.

Articles were selected that reported results of randomized controlled clinical trials of depressive disorders of at least 3 week's duration in which MOC or BRO were contrasted against either $\mathrm{PBO}$ or another antidepressant. Articles were excluded if they presented data from studies previously published or if there was obvious overlap of subjects included in the published reports (e.g., Bakish et al 1992b, c; Lonnqvist et al. 1994a, b; Nair et al. 1995a, b). Four classes of comparisons were reported: separate contrasts of MOC and $B R O$ versus PBO and separate contrasts of MOC and BRO versus active antidepressants. No study was found that directly contrasted the antidepressant effects of MOC and BRO.

\section{Evidence Tables}

Each article meeting inclusion criteria was abstracted and summarized on evidence tables (see Table 1 for $\mathrm{BRO}$ and Table 2 for MOC). The evidence tables summarize the following: categorical outcomes for each of the studied drugs, number of patients randomized to and completing each treatment cell, and associated attrition rates. In a number of studies, the exact number of subjects lost to attrition was not specified.
We initially intended to focus our review on peerreviewed publications. However, a majority of the relevant articles were found to be published in journal supplements. The review process employed for such supplements is quite variable and, as the costs of printing and distribution of supplements are typically supported by the manufacturer of the antidepressant, the potential for bias should not be overlooked. Nevertheless, we concluded that it would be more informative to include these studies than to exclude them.

As in our earlier report of the older MAOIs (Thase et al. 1995), response was defined as the percentage of patients achieving either a 50\% reduction in Hamilton Rating Scale for Depression (Hamilton 1960) or a final Clinical Global Impression (Guy 1976) score of 1 or 2 (markedly improved or very much improved). These definitions were chosen because they are the most commonly reported methods used to determine treatment response rates in randomized clinical trials (Angst et al. 1993; Prien et al. 1991; Thase and Kupfer 1987). This method of determining outcome includes a number of partially remitted cases (i.e., patients with significant residual symptomatology). Different results might have emerged if more stringent definitions of outcome, such as complete remission (e.g., 17-item Hamilton Scale $\leqslant 7$ were used. However, very few studies report remission rates, and there is also no reason to assume that use of remission criterian would have advantaged one class of drugs over the other (e.g., Angst et al. 1993). When looking across acute phase stud-

Table 1. Controlled Trials of Brofaromine (BRO) in Depression

\begin{tabular}{|c|c|c|c|c|c|}
\hline \multirow[b]{2}{*}{ Author } & \multirow[b]{2}{*}{ Duration } & \multirow[b]{2}{*}{ Rx Cells (Daily Dosages) } & \multirow{2}{*}{$\begin{array}{l}\text { Rand }(n) \\
\text { Comp }(n)\end{array}$} & \multicolumn{2}{|c|}{ Hamilton } \\
\hline & & & & Pre & Post \\
\hline \multirow[t]{2}{*}{ Möller and Wendt (1989) } & \multirow[t]{2}{*}{8 weeks } & $\mathrm{BRO}$ & $165 / 138$ & NR & NR \\
\hline & & IMI & $82 / 63$ & NR & NR \\
\hline \multirow[t]{2}{*}{ Möller and Volz (1992) } & \multirow[t]{2}{*}{8 weeks } & BRO (93.1 mg) & $160 / 144$ & 29.4 & $9^{*}$ \\
\hline & & IMI (92 mg) & $80 / 72$ & 28.2 & $12^{*}$ \\
\hline \multirow[t]{2}{*}{ Celada et al. (1992) } & \multirow[t]{2}{*}{6 weeks } & $\mathrm{BRO}(150 \mathrm{mg})$ & 8 & 25 & 14.3 \\
\hline & & Phen $(45 \mathrm{mg})$ & 9 & 25.3 & 8.5 \\
\hline \multirow[t]{2}{*}{ Möller and Volz (1993) } & \multirow[t]{2}{*}{8 weeks } & BRO (100-150 mg) & $127 / 100$ & 26.3 & 12.2 \\
\hline & & IMI (100-150 mg) & $62 / 49$ & 26 & 12.6 \\
\hline \multirow[t]{2}{*}{ Hoencamp et al. (1994) } & \multirow[t]{2}{*}{6 weeks } & $\mathrm{BRO}(150 \mathrm{mg})$ & $25 / 21$ & 19.4 & 15.2 \\
\hline & & $\mathrm{MAO}+\mathrm{Li}(75-200 \mathrm{mg} ; 0.6-1.0 \mathrm{mEq} / 1)$ & $26 / 21$ & 19 & 13.4 \\
\hline \multirow[t]{2}{*}{ Nolen et al. (1993) } & \multirow[t]{2}{*}{4 weeks } & $\mathrm{BRO}(218 \mathrm{mg})$ & $22 / 22$ & 26 & $18^{*}$ \\
\hline & & TCP (84 mg) & $17 / 15$ & 27 & $17^{*}$ \\
\hline \multirow[t]{2}{*}{ Volz et al. (1994a) } & \multirow[t]{2}{*}{6 weeks } & BRO (100-150 mg) & $46 / 42$ & 27.9 & 12.4 \\
\hline & & TCP $(20-30 \mathrm{mg})$ & $47 / 43$ & 27.1 & 11.8 \\
\hline \multirow[t]{6}{*}{ Volz et al. (1994b) } & \multirow[t]{6}{*}{4 weeks } & BRO 50 & $13 / 12$ & 22.5 & $10^{*}$ \\
\hline & & BRO 100 & $12 / 12$ & 27.0 & $14^{*}$ \\
\hline & & BRO 150 & $11 / 10$ & 24.9 & $12^{*}$ \\
\hline & & TCP (20 mg) & $11 / 10$ & 25.2 & $12^{*}$ \\
\hline & & & Total $35 / 31$ & & \\
\hline & & & $11 / 8$ & & \\
\hline \multirow[t]{2}{*}{ Chouinard et al. (1993) } & \multirow[t]{2}{*}{6 weeks } & BRO (150 mg) & $111 / 68$ & 25.2 & 15.4 \\
\hline & & $\mathrm{PBO}$ & $109 / 57$ & 24.5 & 16.2 \\
\hline
\end{tabular}

${ }^{*}$ Data estimated from published figure. 
Table 2. Controlled Trials of Moclobemide (MOC) in Depression

\begin{tabular}{|c|c|c|c|c|c|}
\hline \multirow[b]{2}{*}{ Author } & \multirow[b]{2}{*}{ Duration } & \multirow{2}{*}{$\begin{array}{l}\text { Rx Cells } \\
\text { (Dosages) }\end{array}$} & \multirow{2}{*}{$\begin{array}{l}\text { Randomized }(n) / \\
\text { Completers }(n)\end{array}$} & \multicolumn{2}{|c|}{ Hamilton } \\
\hline & & & & Pre & Post \\
\hline \multirow[t]{2}{*}{ Larsen et al. (1984) } & \multirow[t]{2}{*}{6 weeks } & $\begin{array}{l}\text { Moclobemide } \\
\quad(100-300 \mathrm{mg})\end{array}$ & $19 / 13$ & 17.5 & 8.5 \\
\hline & & $\begin{array}{l}\text { Clomipramine } \\
(75-150 \mathrm{mg})\end{array}$ & $19 / 16$ & 19.5 & 9.2 \\
\hline \multirow[t]{2}{*}{ Casacchia et al. (1984) } & \multirow[t]{2}{*}{4 weeks } & $\begin{array}{l}\text { Moclobemide } \\
(297 \mathrm{mg} / \mathrm{d})\end{array}$ & $18 / 13$ & 41.7 & 16.5 \\
\hline & & Placebo & $16 / 7$ & 39.3 & 29.1 \\
\hline \multirow[t]{2}{*}{ Stefanis et al. (1984) } & & Moclobemide & & NR & NR \\
\hline & & Desipramine & Total: 49 & NR & NR \\
\hline \multirow[t]{2}{*}{ Norman et al. (1985) } & \multirow[t]{2}{*}{4 weeks } & $\begin{array}{l}\text { Moclobemide } \\
\quad(150-450 \mathrm{mg})\end{array}$ & $13 / 13$ & 26.1 & 17.2 \\
\hline & & $\begin{array}{l}\text { Amitiyptyline } \\
\quad(150-250 \mathrm{mg})\end{array}$ & $12 / 12$ & 25.4 & 16.0 \\
\hline \multirow[t]{2}{*}{ Koczkas et al. (1989) } & \multirow[t]{2}{*}{6 weeks } & $\begin{array}{l}\text { Moclobemide } \\
\text { (300 mg) }\end{array}$ & $32 / 19$ & 22.3 & 6.3 \\
\hline & & $\begin{array}{l}\text { Clomipramine } \\
\quad(150 \mathrm{mg})\end{array}$ & $30 / 21$ & 22.8 & 7.3 \\
\hline \multirow[t]{3}{*}{ Versiani et al. (1989) } & \multirow[t]{3}{*}{6 weeks } & $\begin{array}{l}\text { Moclobemide } \\
\quad(300-600 \mathrm{mg})\end{array}$ & $164 / 138$ & 26.0 & 12.0 \\
\hline & & $\begin{array}{l}\text { Imipramine } \\
\quad(33.3-200 \mathrm{mg})\end{array}$ & $164 / 135$ & 25.5 & 11.3 \\
\hline & & Placebo & $162 / 126$ & 25.4 & 20.9 \\
\hline \multirow[t]{2}{*}{ Casacchia and Rossi (1989) } & \multirow[t]{2}{*}{4 weeks } & $\begin{array}{l}\text { Moclobemide } \\
\quad(159-300 \mathrm{mg})\end{array}$ & $20 / 16$ & 32.4 & 10.7 \\
\hline & & $\begin{array}{l}\text { Imipramine } \\
\quad(75-150 \mathrm{mg})\end{array}$ & $20 / 15$ & 32.3 & 11.4 \\
\hline \multirow[t]{2}{*}{ Baumhackl et al. (1989) } & \multirow[t]{2}{*}{4 weeks } & $\begin{array}{l}\text { Moclobemide } \\
\quad(300-600 \mathrm{mg})\end{array}$ & $189 / 154$ & 25.0 & $10.0^{*}$ \\
\hline & & $\begin{array}{l}\text { Imipramine } \\
\quad(100-200 \mathrm{mg})\end{array}$ & 192/171 & 24.3 & $11.0^{*}$ \\
\hline \multirow[t]{2}{*}{ Tiller et al. (1989) } & \multirow[t]{2}{*}{8 weeks } & $\begin{array}{l}\text { Moclobemide } \\
\quad(150-450 \mathrm{mg})\end{array}$ & $20 / 14$ & 23.0 & 11.3 \\
\hline & & $\begin{array}{l}\text { Diazepam } \\
\quad(15-45 \mathrm{mg})\end{array}$ & $20 / 14$ & 22.1 & 6.1 \\
\hline \multirow[t]{3}{*}{ Larsen et al. (1989) } & \multirow[t]{3}{*}{6 weeks } & $\begin{array}{l}\text { Moclobemide } \\
\quad(300 \mathrm{mg})\end{array}$ & $22 / 16$ & 17.5 & 10.5 \\
\hline & & $\begin{array}{l}\text { Clomipramine } \\
\quad(150 \mathrm{mg})\end{array}$ & $20 / 17$ & 17.8 & 7.5 \\
\hline & & Placebo & $18 / 13$ & 18.3 & 12.5 \\
\hline \multirow[t]{2}{*}{ Newburn et al. (1990) } & \multirow[t]{2}{*}{6 weeks } & $\begin{array}{l}\text { Moclobemide } \\
\quad(200-400 \mathrm{mg})\end{array}$ & $26 / 21$ & $28.0^{*}$ & $7.0^{*}$ \\
\hline & & $\begin{array}{l}\text { Amitriptyline } \\
\quad(125-150 \mathrm{mg})\end{array}$ & $23 / 16$ & $27.0^{*}$ & $5.0^{*}$ \\
\hline \multirow[t]{2}{*}{ Gabelic and Kuhn (1990) } & \multirow[t]{2}{*}{4 weeks } & $\begin{array}{l}\text { Moclobemide } \\
\quad(100-350 \mathrm{mg})\end{array}$ & $20 / N R$ & NR & NR \\
\hline & & $\begin{array}{l}\text { Tranylcypromine } \\
\quad(10-30 \mathrm{mg})\end{array}$ & $20 / \mathrm{NR}$ & NR & NR \\
\hline \multirow[t]{2}{*}{ DeVanna et al. (1990) } & 4 weeks & $\begin{array}{l}\text { Moclobemide } \\
\quad(300-500 \mathrm{mg})\end{array}$ & $40 / 32$ & 23.4 & 11.1 \\
\hline & & $\begin{array}{l}\text { Mianserin } \\
\quad(75-125 \mathrm{mg})\end{array}$ & $40 / 34$ & 22.5 & 10.0 \\
\hline DeVanna et al. (1990) & 6 weeks & $\begin{array}{l}\text { Moclobemide } \\
\quad(150-300 \mathrm{mg})\end{array}$ & NR & 33.4 & 5.1 \\
\hline & & $\begin{array}{l}\text { Maprotiline } \\
\quad(75-150 \mathrm{mg})\end{array}$ & $\begin{array}{l}\text { NR } \\
\text { Total } 39\end{array}$ & 29.4 & 5.1 \\
\hline Casacchia and Moll (1990) & 4 weeks & $\begin{array}{l}\text { Moclobemide } \\
\quad(100-300 \mathrm{mg})\end{array}$ & $20 / \mathrm{NR}$ & NR & NR \\
\hline & & Imipramine & $20 / \mathrm{NR}$ & NR & NR \\
\hline
\end{tabular}


Table 2. (continued)

\begin{tabular}{|c|c|c|c|c|c|}
\hline \multirow[b]{2}{*}{ Author } & \multirow[b]{2}{*}{ Duration } & \multirow{2}{*}{$\begin{array}{l}\text { Rx Cells } \\
\text { (Dosages) }\end{array}$} & \multirow{2}{*}{$\begin{array}{l}\text { Randomized }(n) / \\
\text { Completers }(n)\end{array}$} & \multicolumn{2}{|c|}{ Hamilton } \\
\hline & & & & Pre & Post \\
\hline \multirow[t]{2}{*}{ Classen and Laux (1990) } & \multirow[t]{2}{*}{4 weeks } & Moclobemide & $20 / 13$ & 28.3 & 14.4 \\
\hline & & Maprotiline & $20 / 18$ & 28.1 & 15.9 \\
\hline \multirow[t]{2}{*}{ Beckers et al. (1990) } & \multirow[t]{2}{*}{4 weeks } & $\begin{array}{l}\text { Moclobemide } \\
\quad(300-328 \mathrm{mg})\end{array}$ & $8 / \mathrm{NR}$ & $26.0^{*}$ & $5.0^{*}$ \\
\hline & & $\begin{array}{l}\text { Amitiyptyline } \\
\text { (75-96 mg) }\end{array}$ & $9 / \mathrm{NR}$ & $27.0^{*}$ & $8.0^{*}$ \\
\hline \multirow[t]{2}{*}{ Beckers et al. (1990) } & \multirow[t]{2}{*}{4 weeks } & $\begin{array}{l}\text { Moclobemide } \\
\quad(294-408 \mathrm{mg})\end{array}$ & 13/NA & $24.0^{*}$ & $11.0^{*}$ \\
\hline & & $\begin{array}{l}\text { Amitriptyline } \\
(95-129 \mathrm{mg})\end{array}$ & 14/NA & $30.0^{*}$ & $12.0^{*}$ \\
\hline \multirow[t]{2}{*}{ Dierick et al. (1990) } & \multirow[t]{2}{*}{4 weeks } & $\begin{array}{l}\text { Moclobemide } \\
\quad(300-600 \mathrm{mg})\end{array}$ & $32 / 29$ & $24.0^{*}$ & $13.0^{*}$ \\
\hline & & $\begin{array}{l}\text { Clomipramine } \\
(75-150 \mathrm{mg})\end{array}$ & $31 / 24$ & $23.0^{*}$ & $12.0^{*}$ \\
\hline \multirow[t]{2}{*}{ Civeira (1990) } & \multirow[t]{2}{*}{4 weeks } & $\begin{array}{l}\text { Moclobemide } \\
\quad(150 \mathrm{mg})\end{array}$ & $33 / 27$ & $30.0^{*}$ & $8.0^{*}$ \\
\hline & & $\begin{array}{l}\text { Clomipramine } \\
\text { (75 mg) }\end{array}$ & $31 / 26$ & $32.0^{*}$ & $9.0^{*}$ \\
\hline \multirow{2}{*}{$\begin{array}{l}\text { Gabelic and Moll (1990) } \\
\text { (Not clear if ITT or AT } \\
\text { used for calculation) }\end{array}$} & \multirow[t]{2}{*}{4 weeks } & $\begin{array}{l}\text { Moclobemide } \\
\quad(100-250 \mathrm{mg})\end{array}$ & $15 / 14$ & NR & NR \\
\hline & & $\begin{array}{l}\text { Desipramine } \\
\quad(50-150 \mathrm{mg})\end{array}$ & $15 / 9$ & NR & NR \\
\hline \multirow{3}{*}{$\begin{array}{l}\text { Biziere and Berger (1990) } \\
\text { (See Versiani and } \\
\text { Baumhackl) }\end{array}$} & \multirow[t]{3}{*}{6 weeks } & $\begin{array}{l}\text { Moclobemide } \\
\quad(300-600 \mathrm{mg})\end{array}$ & $164 / 146$ & NR & NR \\
\hline & & $\begin{array}{l}\text { Imipramine } \\
\quad(100-200 \mathrm{mg})\end{array}$ & $164 / 145$ & NR & NR \\
\hline & & Placebo & $162 / 120$ & & \\
\hline \multirow[t]{3}{*}{$\begin{array}{l}\text { Lecrubier and Guelfi } \\
\text { (1990) }\end{array}$} & \multirow[t]{3}{*}{6 weeks } & $\begin{array}{l}\text { Moclobemide } \\
\quad(300-600 \mathrm{mg})\end{array}$ & $164 / 146$ & $26.0^{*}$ & $10.0^{*}$ \\
\hline & & $\begin{array}{l}\text { Imipramine } \\
\quad(100-200 \mathrm{mg})\end{array}$ & $164 / 145$ & $26.0^{*}$ & $10.0^{*}$ \\
\hline & & Placebo & $162 / 120$ & & \\
\hline \multirow[t]{2}{*}{$\begin{array}{l}\text { Lecrubier and Guelfi } \\
\text { (1990) }\end{array}$} & \multirow[t]{2}{*}{6 weeks } & $\begin{array}{l}\text { Moclobemide } \\
\quad(300-600 \mathrm{mg})\end{array}$ & $62 / 47(24 \%)$ & $35.0^{*}$ & 10.0 \\
\hline & & $\begin{array}{l}\text { Clomipramine } \\
\quad(100-200 \mathrm{mg})\end{array}$ & $67 / 56(16 \%)$ & 35.0 & $10.0^{*}$ \\
\hline \multirow[t]{2}{*}{$\begin{array}{l}\text { Lecrubier and Guelfi } \\
\text { (1990) }\end{array}$} & \multirow[t]{2}{*}{12 weeks } & $\begin{array}{l}\text { Moclobemide } \\
\quad(400-600 \mathrm{mg})\end{array}$ & $\begin{array}{l}\text { 98/74 }(12 \mathrm{w}) \\
\text { NR for } 6 \text { weeks }\end{array}$ & $25.0^{*}$ & $10.0^{*}$ \\
\hline & & $\begin{array}{l}\text { Clomipramine } \\
\quad(100-150 \mathrm{mg})\end{array}$ & $\begin{array}{l}\text { 93/65 (12 w) } \\
\text { NR for } 6 \text { weeks }\end{array}$ & $25.0^{*}$ & $10.0^{*}$ \\
\hline \multirow[t]{3}{*}{ Ucha Udabe et al. (1990) } & \multirow[t]{3}{*}{6 weeks } & $\begin{array}{l}\text { Moclobemide } \\
\quad(300-600 \mathrm{mg})\end{array}$ & $24 / \mathrm{NR}$ & $21.0^{*}$ & $10.0^{*}$ \\
\hline & & $\begin{array}{l}\text { Imipramine } \\
\quad(33.3-200 \mathrm{mg})\end{array}$ & $24 / \mathrm{NR}$ & $21.0^{*}$ & $10.0^{*}$ \\
\hline & & Placebo & $24 / N R$ & & \\
\hline \multirow[t]{3}{*}{ Versiani et al. (1990) } & \multirow[t]{3}{*}{6 weeks } & $\begin{array}{l}\text { Moclobemide } \\
\quad(300-600 \mathrm{mg})\end{array}$ & $25 / 20$ & $25.0^{*}$ & $5.0^{*}$ \\
\hline & & $\begin{array}{l}\text { Imipramine } \\
\quad(33-200 \mathrm{mg})\end{array}$ & $25 / 22$ & $25.0^{*}$ & $5.0^{*}$ \\
\hline & & Placebo & $25 / 22$ & $23.0^{*}$ & $8.0^{*}$ \\
\hline Rossel and Moll (1990) & 4 weeks & $\begin{array}{l}\text { Moclobemide } \\
\quad(150-300 \mathrm{mg})\end{array}$ & $23 / \mathrm{NR}$ & NR & NR \\
\hline & & $\begin{array}{l}\text { Tranylcypromine } \\
\quad(15-30 \mathrm{mg})\end{array}$ & $24 / N R$ & NR & NR \\
\hline Botte et al. (1990) & 4 weeks & $\begin{array}{l}\text { Moclobemide } \\
\quad(300-600 \mathrm{mg})\end{array}$ & $20 / N R$ & NR & NR \\
\hline & & $\begin{array}{l}\text { Tranylcypromine } \\
\quad(15-30 \mathrm{mg})\end{array}$ & $20 / N R$ & NR & NR \\
\hline
\end{tabular}


Table 2. (continued)

\begin{tabular}{|c|c|c|c|c|c|}
\hline \multirow[b]{2}{*}{ Author } & \multirow[b]{2}{*}{ Duration } & \multirow{2}{*}{$\begin{array}{l}\text { Rx Cells } \\
\text { (Dosages) }\end{array}$} & \multirow{2}{*}{$\begin{array}{l}\text { Randomized }(n) / \\
\text { Completers }(n)\end{array}$} & \multicolumn{2}{|c|}{ Hamilton } \\
\hline & & & & Pre & Post \\
\hline \multirow[t]{2}{*}{ Funke et al. (1990) } & \multirow[t]{2}{*}{4 weeks } & $\begin{array}{l}\text { Moclobemide } \\
\text { (mean 18-346 } \\
\text { mg) }\end{array}$ & $15 / 14$ & $23.0^{*}$ & $10.0^{*}$ \\
\hline & & $\begin{array}{l}\text { Clomipramine } \\
\text { (mean max } 183 \\
\text { mg) }\end{array}$ & $15 / 15$ & $22.5^{*}$ & $10.0^{*}$ \\
\hline \multirow[t]{2}{*}{ Tiller et al. (1990) } & \multirow[t]{2}{*}{8 weeks } & $\begin{array}{l}\text { Moclobemide } \\
\quad(150-600 \mathrm{mg})\end{array}$ & $13 / 11$ & 15.0 & NR \\
\hline & & $\begin{array}{l}\text { Mianserin } \\
\quad(30-90 \mathrm{mg})\end{array}$ & $\begin{array}{l}\text { 13/9 (4 week } \\
\text { data were used } \\
\text { for calculation) }\end{array}$ & 16.8 & NR \\
\hline \multirow[t]{3}{*}{ Larsen et al. (1991) } & \multirow[t]{5}{*}{6 weeks } & $\begin{array}{l}\text { Moclobemide } \\
\quad(300-600 \mathrm{mg})\end{array}$ & $59 / 41$ & 20.9 & 10.2 \\
\hline & & $\begin{array}{l}\text { Clomipramine } \\
\quad(150-200 \mathrm{mg})\end{array}$ & $57 / 39$ & 20.2 & 5.9 \\
\hline & & $\begin{array}{l}\text { Isocarboxide } \\
\quad(30-40 \mathrm{mg})\end{array}$ & $51 / 39$ & 21.4 & 8.3 \\
\hline \multirow[t]{2}{*}{ Barrelet et al (1991) } & & $\begin{array}{l}\text { Moclobemide } \\
\quad(300-450 \mathrm{mg})\end{array}$ & NR & NR & NR \\
\hline & & $\begin{array}{l}\text { Fluvoxamine } \\
\quad(100-200 \mathrm{mg})\end{array}$ & NR & NR & NR \\
\hline \multirow[t]{2}{*}{ Guelfi et al. (1992) } & \multirow[t]{2}{*}{6 weeks } & $\begin{array}{l}\text { Moclobemide } \\
\quad(75-450 \mathrm{mg})\end{array}$ & $62 / 47$ & 27.3 & 11.1 \\
\hline & & $\begin{array}{l}\text { Clomipramine } \\
(25-150 \mathrm{mg})\end{array}$ & $67 / 56$ & 27.7 & 9.6 \\
\hline \multirow[t]{3}{*}{ Bakish et al. (1992c) } & \multirow[t]{3}{*}{6 weeks } & $\begin{array}{l}\text { Moclobemide } \\
\quad(200-600 \mathrm{mg})\end{array}$ & $18 / 15$ & 22.9 & 10.9 \\
\hline & & $\begin{array}{l}\text { Amitryptiline } \\
\text { (50-150 mg) }\end{array}$ & $19 / 9$ & 22.4 & 12.4 \\
\hline & & Placebo & $18 / 8$ & 23.4 & 16.6 \\
\hline \multirow[t]{3}{*}{ Bakish et al. (1992b) } & \multirow[t]{3}{*}{6 weeks } & $\begin{array}{l}\text { Moclobemide } \\
\quad(200-600 \mathrm{mg})\end{array}$ & $57 / 41$ & 23.8 & NR \\
\hline & & $\begin{array}{l}\text { Amitriptyline } \\
\text { (50-150 mg) }\end{array}$ & $57 / 41$ & 22.8 & NR \\
\hline & & Placebo & $55 / 29$ & 23.0 & NR \\
\hline \multirow[t]{2}{*}{ Bougerol et al. (1992) } & \multirow[t]{2}{*}{4 weeks } & $\begin{array}{l}\text { Moclobemide } \\
\quad(150-450 \mathrm{mg})\end{array}$ & $65 / 50$ & 24.4 & 8.5 \\
\hline & & $\begin{array}{l}\text { Fluvoxamine } \\
\quad(50-200 \mathrm{mg})\end{array}$ & $61 / 42$ & 25.1 & 9.1 \\
\hline \multirow[t]{2}{*}{ Ose and Holm (1992) } & \multirow[t]{2}{*}{4 weeks } & $\begin{array}{l}\text { Moclobemide } \\
\quad(300-500 \mathrm{mg})\end{array}$ & $35 / 19$ & $21.0^{*}$ & $12.0^{*}$ \\
\hline & & Placebo & $33 / 21$ & $21.0^{*}$ & $15.0^{*}$ \\
\hline \multirow[t]{2}{*}{$\begin{array}{l}\text { Macher and Mirabaud } \\
\text { (1992) }\end{array}$} & \multirow[t]{2}{*}{4 weeks } & $\begin{array}{l}\text { Moclobemide } \\
\quad(450 \mathrm{mg})\end{array}$ & $90 / 79$ & NR & NR \\
\hline & & $\begin{array}{l}\text { Amineptine } \\
\quad(200 \mathrm{mg})\end{array}$ & $94 / 81$ & NR & NR \\
\hline \multirow[t]{3}{*}{ Allain et al. (1992) } & \multirow[t]{3}{*}{6 weeks } & $\begin{array}{l}\text { Moclobemide } \\
\quad(300-450 \mathrm{mg})\end{array}$ & NA/13 & NR & NR \\
\hline & & $\begin{array}{l}\text { Maprotiline } \\
\quad(100-150 \mathrm{mg})\end{array}$ & NA/12 & NR & NR \\
\hline & & $\begin{array}{l}\text { Viloxazine } \\
\quad(200-300 \mathrm{mg})\end{array}$ & $\begin{array}{l}\text { NA/12 } \\
\text { Total } 46 / 37\end{array}$ & NR & NR \\
\hline \multirow[t]{2}{*}{ Evans et al. (1992) } & \multirow[t]{2}{*}{4 weeks } & $\begin{array}{l}\text { Moclobemide } \\
\quad(300-750 \mathrm{mg})\end{array}$ & $24 / 19$ & 23.9 & 12.1 \\
\hline & & $\begin{array}{l}\text { Imipramine } \\
\quad(100-250 \mathrm{mg})\end{array}$ & $24 / 17$ & 23.1 & 9.4 \\
\hline \multirow[t]{2}{*}{ Versiani et al. (1992) } & \multirow[t]{2}{*}{8 weeks } & $\begin{array}{l}\text { Moclobemide } \\
\quad(300-750 \mathrm{mg})\end{array}$ & NR & NR & NR \\
\hline & & $\begin{array}{l}\text { Imipramine } \\
\quad(100-250 \mathrm{mg})\end{array}$ & $\begin{array}{l}\text { NR } \\
\text { Total } 315\end{array}$ & NR & NR \\
\hline
\end{tabular}


Table 2. (continued)

\begin{tabular}{|c|c|c|c|c|c|}
\hline \multirow[b]{2}{*}{ Author } & \multirow[b]{2}{*}{ Duration } & \multirow{2}{*}{$\begin{array}{l}\text { Rx Cells } \\
\text { (Dosages) }\end{array}$} & \multirow{2}{*}{$\begin{array}{l}\text { Randomized }(n) / \\
\text { Completers }(n)\end{array}$} & \multicolumn{2}{|c|}{ Hamilton } \\
\hline & & & & Pre & Post \\
\hline \multirow[t]{2}{*}{$\begin{array}{l}\text { Lemoine and Mirabaud } \\
\text { (1992) }\end{array}$} & 4 weeks & $\begin{array}{l}\text { Moclobemide } \\
\quad(450 \mathrm{mg})\end{array}$ & $135 / \mathrm{NR}$ & & \\
\hline & & $\begin{array}{l}\text { Toloxatone } \\
\text { (1000 mg) }\end{array}$ & $133 / \mathrm{NR}$ & & \\
\hline \multirow[t]{2}{*}{ Lingjaerde et al. (1993) } & 3 weeks & $\begin{array}{l}\text { Moclobemide } \\
\quad(400 \mathrm{mg})\end{array}$ & $16 / N R$ & $38^{*}$ & $24^{*}$ \\
\hline & & Placebo & $18 / \mathrm{NR}$ & $32^{*}$ & $21^{*}$ \\
\hline \multirow[t]{2}{*}{ Williams et al. (1993) } & 6 weeks & $\begin{array}{l}\text { Moclobemide } \\
\quad(300-600 \mathrm{mg})\end{array}$ & $62 / 47$ & 24.0 & 5.0 \\
\hline & & $\begin{array}{l}\text { Fluoxetine } \\
\quad(20-40 \mathrm{mg})\end{array}$ & $60 / 45$ & 24.0 & 11.0 \\
\hline \multirow[t]{2}{*}{ Bocksberger et al. (1993) } & 4 weeks & $\begin{array}{l}\text { Moclobemide } \\
\quad(300-450 \mathrm{mg})\end{array}$ & $20 / 19$ & 41.9 & 11.9 \\
\hline & & $\begin{array}{l}\text { Fluvoxamine } \\
\quad(100-200 \mathrm{mg})\end{array}$ & $20 / 18$ & 43.3 & 23.5 \\
\hline \multirow[t]{2}{*}{ Steinmeyer et al. (1993) } & 6 weeks & $\begin{array}{l}\text { Moclobemide } \\
\quad(300-600 \mathrm{mg})\end{array}$ & $58 / 52$ & 25.5 & 10.0 \\
\hline & & $\begin{array}{l}\text { Maprotiline } \\
\quad(75-150 \mathrm{mg})\end{array}$ & $57 / 53$ & 24.8 & 8.5 \\
\hline \multirow[t]{2}{*}{ Heinze et al. (1993) } & 4 weeks & $\begin{array}{l}\text { Moclobemide } \\
\quad(100-300 \mathrm{mg})\end{array}$ & $81 / 80$ & 29.1 & 29.4 \\
\hline & & $\begin{array}{l}\text { Tranylocypromine } \\
\quad(10-30 \mathrm{mg})\end{array}$ & $79 / 77$ & 9.9 & 9.5 \\
\hline \multirow[t]{2}{*}{ Danish U.A.G. (1993) } & 6 weeks & $\begin{array}{l}\text { Moclobemide } \\
\quad(400 \mathrm{mg})\end{array}$ & $57 / 51$ & $24^{*}$ & $15^{*}$ \\
\hline & & $\begin{array}{l}\text { Clomipramine } \\
\quad(150 \mathrm{mg})\end{array}$ & $58 / 56$ & $24^{*}$ & $11^{*}$ \\
\hline \multirow[t]{2}{*}{ Rimon et al. (1993) } & 4 weeks & $\begin{array}{l}\text { Moclobemide } \\
\quad(150-525 \mathrm{mg})\end{array}$ & $62 / 55$ & 22.6 & 8.3 \\
\hline & & $\begin{array}{l}\text { Imipramine } \\
(50-175 \mathrm{mg})\end{array}$ & $65 / 58$ & 22.1 & $8.9^{*}$ \\
\hline \multirow[t]{2}{*}{ Philipp et al. (1993) } & 6 weeks & $\begin{array}{l}\text { Moclobemide } \\
\quad(400 \mathrm{mg})\end{array}$ & $118 / 94$ & NR & NR \\
\hline & & $\begin{array}{l}\text { Doxepine } \\
\quad(100 \mathrm{mg})\end{array}$ & $119 / 89^{*}$ & NR & NR \\
\hline \multirow[t]{2}{*}{ Beaumont et al. (1993) } & 6 weeks & $\begin{array}{l}\text { Moclobemide } \\
\quad(450 \mathrm{mg})\end{array}$ & $170 / 132$ & 21.4 & 11.1 \\
\hline & & $\begin{array}{l}\text { Dothiepin } \\
\quad(75-150 \mathrm{mg})\end{array}$ & $175 / 133$ & 21.2 & 8.5 \\
\hline \multirow[t]{3}{*}{ Kusalic et al. (1993) } & 6 weeks & $\begin{array}{l}\text { Moclobemide } \\
\quad(482.6)\end{array}$ & $\mathrm{NR} / 11$ & NR & NR \\
\hline & & $\begin{array}{l}\text { Amitriptyline } \\
(109.93)\end{array}$ & $\mathrm{NR} / 13$ & NR & NR \\
\hline & & Placebo & $\mathrm{NR} / 15$ & NR & NR \\
\hline \multirow[t]{2}{*}{ Lonnqvist et al. (1994a) } & 6 weeks & $\begin{array}{l}\text { Moclobemide } \\
\quad(300-450 \mathrm{mg})\end{array}$ & $24 / 21$ & 22.9 & 9.2 \\
\hline & & $\begin{array}{l}\text { Fluoxetine } \\
\quad(20-40 \mathrm{mg})\end{array}$ & $29 / 20$ & 21.8 & 10.6 \\
\hline \multirow[t]{2}{*}{ Lonnqvist et al. (1994b) } & 6 weeks & $\begin{array}{l}\text { Moclobemide } \\
\quad(300-450 \mathrm{mg})\end{array}$ & $102 / 84$ & 24.4 & 10.0 \\
\hline & & $\begin{array}{l}\text { Fluoxetine } \\
\quad(20-40 \mathrm{mg})\end{array}$ & $107 / 85$ & 24.6 & 11.0 \\
\hline \multirow[t]{3}{*}{ UK Study Group (1994) } & 6 weeks & $\begin{array}{l}\text { Moclobemide } \\
\quad(300-450 \mathrm{mg})\end{array}$ & $\mathrm{NR} / 56$ & 24.9 & 13.0 \\
\hline & & $\begin{array}{l}\text { Imipramine } \\
\quad(75-150 \mathrm{mg})\end{array}$ & $\mathrm{RN} / 50$ & 25.4 & 13.5 \\
\hline & & Placebo & $\begin{array}{l}\text { NR/57 } \\
\text { Total: } 249\end{array}$ & 24.4 & 13.8 \\
\hline
\end{tabular}

(continued) 
Table 2. (continued)

\begin{tabular}{|c|c|c|c|c|c|}
\hline \multirow[b]{2}{*}{ Author } & \multirow[b]{2}{*}{ Duration } & \multirow{2}{*}{$\begin{array}{l}\text { Rx Cells } \\
\text { (Dosages) }\end{array}$} & \multirow{2}{*}{$\begin{array}{l}\text { Randomized }(n) / \\
\text { Completers }(n)\end{array}$} & \multicolumn{2}{|c|}{ Hamilton } \\
\hline & & & & Pre & Post \\
\hline \multirow[t]{2}{*}{ Geertz et al. (1995) } & \multirow[t]{2}{*}{6 weeks } & $\begin{array}{l}\text { Moclobemide } \\
\quad(300-600 \mathrm{mg})\end{array}$ & $24 / 15$ & 19.7 & 9.1 \\
\hline & & $\begin{array}{l}\text { Fluoxetine } \\
\quad(20-40 \mathrm{mg})\end{array}$ & $25 / 13$ & 21.2 & 9.8 \\
\hline \multirow[t]{2}{*}{ Vaz Serra et al. (1994) } & \multirow[t]{2}{*}{6 weeks } & $\begin{array}{l}\text { Moclobemide } \\
\quad(300 \mathrm{mg})\end{array}$ & $40 / 38$ & 23.6 & 9.1 \\
\hline & & $\begin{array}{l}\text { Maprotiline } \\
\text { (75 mg) }\end{array}$ & $40 / 37$ & 25.7 & 9.8 \\
\hline \multirow[t]{2}{*}{ Gachoud et al. (1994) } & \multirow[t]{2}{*}{4 weeks } & $\begin{array}{l}\text { Moclobemide } \\
\quad(300-400 \mathrm{mg})\end{array}$ & $66 / 54$ & 26.5 & 11.9 \\
\hline & & $\begin{array}{l}\text { Maprotiline } \\
\quad(75-150 \mathrm{mg})\end{array}$ & $64 / 55$ & 24.3 & 10.4 \\
\hline \multirow[t]{2}{*}{ Pancheri et al. (1994) } & \multirow[t]{2}{*}{8 weeks } & $\begin{array}{l}\text { Moclobemide } \\
\quad(400-600 \mathrm{mg})\end{array}$ & $15 / 10$ & 27.2 & 13.4 \\
\hline & & $\begin{array}{l}\text { Imipramine } \\
\quad(20-100 \mathrm{mg})\end{array}$ & $15 / 15$ & 24.3 & 13.2 \\
\hline \multirow[t]{3}{*}{ Nair et al. (1995b) } & \multirow[t]{3}{*}{7 weeks } & $\begin{array}{l}\text { Moclobemide } \\
\quad(100-400 \mathrm{mg})\end{array}$ & $36 / 15$ & 23.0 & 11.0 \\
\hline & & $\begin{array}{l}\text { Nortriptyline } \\
\quad(25-100 \mathrm{mg})\end{array}$ & $38 / 20$ & 23.5 & 6.5 \\
\hline & & Placebo & $35 / 20$ & 24.0 & 16.0 \\
\hline \multirow[t]{2}{*}{ Gattaz et al. (1995) } & \multirow[t]{2}{*}{6 weeks } & $\begin{array}{l}\text { Moclobemide } \\
\quad(300-600 \mathrm{mg})\end{array}$ & $36 / 27$ & $28.0^{*}$ & $12.5^{*}$ \\
\hline & & $\begin{array}{l}\text { Fluoxetine } \\
\quad(20-40 \mathrm{mg})\end{array}$ & $34 / 26$ & $28.3^{*}$ & $12.5^{*}$ \\
\hline \multirow[t]{2}{*}{ Stabl et al. (1995) } & \multirow[t]{2}{*}{6 weeks } & $\begin{array}{c}\text { Moclobemide } \\
(450 \mathrm{mg})+ \\
\text { Thioridazine } \\
(100 \mathrm{mg})\end{array}$ & $38 / 32$ & 28.0 & NR \\
\hline & & $\begin{array}{l}\text { Moclobemide } \\
(450 \mathrm{mg})+ \\
\text { Placebo }\end{array}$ & $40 / 31$ & 27.0 & NR \\
\hline \multirow[t]{2}{*}{$\begin{array}{l}\text { Kragh-Sorensen et al. } \\
\quad(1995)\end{array}$} & \multirow[t]{2}{*}{6 weeks } & $\begin{array}{l}\text { Moclobemide } \\
\quad(400 \mathrm{mg})\end{array}$ & $48 / 39$ & NR & NR \\
\hline & & $\begin{array}{l}\text { Clomipramine } \\
\quad(150 \mathrm{mg})\end{array}$ & $48 / 30$ & NR & NR \\
\hline \multirow[t]{2}{*}{ Reynaert et al. (1995) } & \multirow[t]{2}{*}{6 weeks } & $\begin{array}{l}\text { Moclobemide } \\
\quad(300-600 \mathrm{mg})\end{array}$ & $51 / 38$ & 24.1 & 12.2 \\
\hline & & $\begin{array}{l}\text { Fluoxetine } \\
\quad(20-40 \mathrm{mg})\end{array}$ & $50 / 42$ & 22.7 & 12.9 \\
\hline \multirow[t]{2}{*}{ Lingjaerde et al. (1995) } & \multirow[t]{2}{*}{6 weeks } & $\begin{array}{l}\text { Moclobemide } \\
\quad(200-600 \mathrm{mg})\end{array}$ & $30 / 26$ & $13^{*}$ & $7^{*}$ \\
\hline & & $\begin{array}{l}\text { Doxepin } \\
\quad(150-250 \mathrm{mg})\end{array}$ & $23 / 20$ & $14^{*}$ & $5^{*}$ \\
\hline
\end{tabular}

Abbreviation: NR $=$ Not Reported.

${ }^{*}$ Data estimated from published figure.

ies with varying lengths of therapy, a more rigorous definition of remission also is likely to underestimate the drug's efficacy in the shorter studies (i.e., 4 weeks) relative to the longer studies (i.e., 6-8 weeks).

The response rates are first reported using modified "Intent to Treat" (ITT) samples. The denominator for the ITT samples is the number randomized to treatment, whereas the numerator is the number who stayed in treatment and responded. This method must be considered "modified" because, in an ideal ITT analysis, outcome data would be collected at all relevant time points and for all randomized patients (i.e., including drop-outs) (e.g., Lavori, 1992). This ideal is almost never actualized. Nevertheless, we strongly favor the ITT method over the major alternative (i.e., an analysis of patients who completed an adequate treatment trial (AT). We prefer the ITT method because exclusion of dropouts may obscure important differences resulting from differential attrition (Lavori, 1992; Thase et al. 1995). When available, we also report AT outcomes to 
permit comparisons with other meta-analyses of antidepressant efficacy (e.g., Depression Guideline Panel, 1993).

\section{Meta-Analysis}

The Confidence Profile Method of meta-analysis (Eddy et al. 1990) was used to calculate the response rates in each study and to provide summary statistics. This method uses a hierarchical, Baysean random-effects model and calculates the probability distribution to describe results expected if a hypothetical additional study were to be performed. By taking into account the heterogeneity of study results, the CPM depicts the expected range of results that would be expected if pharmacotherapists used similar treatment protocols and treated comparable patients in practice.

Each meta-analysis produces a probability distribution that depicts the likelihood that the parameter of interest falls within any particular range of values. With this probability distribution, the reader can determine the probability that the true effect of treatment is greater than, less than, or equal to any selected value by referencing the observed differences in outcomes in relation to the standard deviation of the difference scores. This exact methodology was used in the meta-analysis conducted by the Depression Guideline Panel (1993) report for the Agency for Health Care Planning and Research, as well as in our earlier review of the older MAOIs (Thase et al. 1995).

One key advantage of the hierarchical Baysean approach is the stability of probability estimates if the metaanalysis is based on large numbers of studies. The random-effects model accounts for among-study variations and random biases in the published studies. However, it cannot account for any systematic biases that occurred across all studies, or bias stemming for the general lack of publications of negative findings in the literature.

\section{RESULTS}

Three hundred fifty-one articles were identified for MOC, including 85 comparing it with either another drug or placebo. Forty seven MOC studies met criteria for inclusion in the meta-analysis. Although eightyeight articles about BRO were identified by the literature search, there were just 20 published reports of drug comparisons, and only 9 of these studies were suitable for the meta-analysis. Overall, the meta-analysis included 3318 patients who began treatment with MOC and 527 with BRO, of which $2077(62.6 \%)$ and 431 $(81.8 \%)$ completed adequate treatment trials.

\section{Brofaromine}

All nine of the controlled studies of BRO used DSM-III or DSM-III-R criteria for entrance in the study. One of these studies enrolled only elderly patients (Möller and Volz, 1993). Among the studies included, only a single trial was placebo-controlled (Chouinard et al. 1993). Thus, a meta-analysis versus PBO could not be conducted on a solitary study.

The Chouinard et al. (1993) study is nevertheless informative. This 6-week randomized clinical trial employed a relatively high fixed dose of BRO: $150 \mathrm{mg} /$ day. Brofaromine was found to be significantly more effective than placebo on several outcome measures, but not on the Hamilton Scale, the principal a priori outcome measure. From a regulatory perspective, this result would thus be counted as a "negative" trial. However, posthoc analyses revealed that the lack of a statistically significant difference between the BRO and PBO groups was attributable to exacerbation of scores on the Hamilton Scale insomnia items in BRO-treated patients (see Volz et al. 1995). It is likely that the high fixed dosage of BRO used in this study inadvertently distorted the results.

The 8 remaining studies used an active comparator. Six studies compared BRO with either imipramine (Tofranil) (Möller and Volz 1992, 1993; Volz and Möller 1994), or tranylcypromine (Nolen et al. 1993; Volz et al. 1994a; Volz et al. 1994b), one compared BRO with the combination of maprotiline (Ludiomil) and lithium (Hoencamp et al. 1994), and one used nomifensine as the comparator (Schiwy et al. 1989).

One study included a small number of inpatients along with a large number of outpatients (Möller and Volz 1993). Four studies included only inpatients (Nolen et al. 1993; Volz and Möller, 1994; Volz et al. 1994a; Volz et al. 1994b). The remaining three studies enrolled only outpatients (Schiwy et al. 1989; Möller and Volz 1992; Hoencamp et al, 1994). Three studies specifically enrolled patients with tricyclic resistant depression (Hoencamp et al. 1994; Nolen et al. 1993; Volz et al. 1994a). Although two studies required that patients have a diagnosis of (unipolar) major depressive disorder (Nolen et al. 1993; Volz et al. 1994b), the remainder enrolled patients with a variety of depressive diagnoses (i.e., bipolar, atypical, dysthymic, and unipolar depressive disorders). (Schiwy et al. 1989; Möller and Volz 1992; Möller and Volz 1993; Hoencamp et al. 1994; Volz et al. 1994a). Brofaromine doses ranged from 50 to $150 \mathrm{mg}$ /day across the eight studies, with two reports specifically addressing BRO dose-response relationships (Schiwy et al. 1989; Volz et al. 1994b).

\section{Efficacy}

Results of the six studies of brofaromine suitable for meta-analysis are summarized on Table 3 . The overall $\mathrm{BRO}$ response rate was $58.6 \%(\mathrm{SD}=6.4 \%)$ in the ITT sample, and $66.7 \%(7.4 \%)$ in the AT sample. A differ- 
ence of $4.8 \%(5.3 \%)$ favoring BRO over the active comparator was observed in the ITT sample, and $0.2 \%(17.6 \%)$ was observed in the AT sample. This pattern of results indicates that BRO has a small advantage over TCAs and the older MAOIs because of better tolerability. The marked variability observed in the AT analysis is also noteworthy, although we could not deduce an explanation for this phenomenon.

Safety. As suggested above, BRO was significantly better tolerated than imipramine in two studies (Möller and Volz 1992; Möller and Volz 1993), and tranylcypromine in two studies (Nolen et al. 1993; Volz et al. 1994b). There were no differences in tolerability observed in three other studies (Schiwy et al. 1989; Volz and Möller, 1994; Volz et al. 1994a). Brofaromine was also generally better tolerated than the combination of maprotiline and lithium, although complaints about sleep problems were again higher in the BRO group (Hoencamp et al. 1994).

\section{Moclobemide}

Overall Meta-Analysis of Efficacy Data versus Active Comparators. The ITT analysis used 47 studies, and 39 studies were included in the AT analysis. Across all studies, the ITT MOC response rate was $58.1 \%$ (2.3\%), with an AT response rate of $67.5 \%(2.3 \%)$. Overall, no difference was found between MOC and the active comparators. The ITT difference was 3.2\% (1.9\%) (MOC > comparators), and the overall AT difference was 1.7\% (1.3\%).

Moclobemide Contrasted with Placebo. Across inpatient and outpatient settings, 12 studies contrasted MOC with PBO (see Table 4). There were 11 studies available for ITT comparisons, and eight for AT comparisons. The ITT MOC response rate was $48.6 \%$ (4.2\%), and the AT response rate was $65.0 \%(4.8 \%)$. A $15.8 \%$ (6.1\%) difference was found in comparison to PBO in the ITT sample. In the AT sample, the advantage favoring MOC was $24.2 \%$ (5.6\%). These studies establish that MOC is an efficacious antidepressant, although the drug-placebo differences somewhat smaller than those observed in our meta-analysis of the older, nonselective MAOIs (Thase et al. 1995; see Figure 1).

Outpatient Studies: MOC versus Active Comparators. A total of 17 exclusively outpatient randomized clinical trials were identified, of which 16 were suitable for the ITT analysis. The Lecrubier and Guelfi (1990) report used two comparators, thus permitting a total of 17 comparisons. Thirteen studies were available for the AT analysis. The ITT response rate of outpatients to MOC was $58.9 \%(2.7 \%)$, and the response rate was $73.2 \%$ (3.5\%) in the AT sample (see Table 5).

Five studies compared MOC with imipramine (Lecrubier and Guelfi 1990; Pancheri et al. 1994; Rimon et al. 1993; Ucha Udabe et al. 1990; Versiani et al. 1989). Three studies used clomipramine (Anafranil) as the comparator (Kragh-Sorensen et al. 1995; Larsen et al. 1991; Lecrubier and Guelfi 1990), two studies each used amitriptyline (Elavil) (Bakish et al. 1992c; Kusalic et al. 1993) or maprotiline (Gachoud et al. 1994; Steinmeyer et al. 1993), and one each compared MOC with isocarboxazid (Marplan) (Larsen et al. 1991), fluvoxamine (Luvox) (Bougerol et al. 1992), amineptine (Macher and Mirabaud 1992), doxepin (Sinequan) (Philipp et al. 1993), or dothiepin (Beaumont et al. 1993). Meta-analysis of

Table 3. Acute Phase Treatment Trials Comparing Brofaromine with Other Drugs in Depressed Patients (Mean/Standard Deviation)

\begin{tabular}{|c|c|c|c|c|c|}
\hline \multirow[b]{2}{*}{ Study } & \multirow[b]{2}{*}{ Drug } & \multicolumn{2}{|c|}{ Efficacy } & \multicolumn{2}{|c|}{$\begin{array}{l}\text { Brofaromine } \\
\text { Versus Drug }\end{array}$} \\
\hline & & ITT & AT & ITT & AT \\
\hline \multirow[t]{2}{*}{ Möller and Volz 1992} & \multirow[t]{2}{*}{ IMI } & 69.8 & 77.5 & 12.4 & 1.3 \\
\hline & & 3.6 & 3.4 & 6.5 & 6.5 \\
\hline \multirow[t]{2}{*}{ Möller and Volz 1993} & \multirow[t]{2}{*}{ IMI } & 69.1 & 87.6 & -1.4 & -1.3 \\
\hline & & 4.0 & 3.2 & 6.9 & 5.4 \\
\hline \multirow[t]{2}{*}{ Hoencamp et al. 1994} & \multirow[t]{2}{*}{$\mathrm{MAP}+\mathrm{LI}$} & 21.1 & 25.0 & -2.9 & -4.5 \\
\hline & & 7.8 & 9.0 & 11.2 & 13.1 \\
\hline \multirow[t]{2}{*}{ Nolen et al. 1993} & \multirow[t]{2}{*}{ TCP } & 45.6 & 45.6 & 15.1 & 11.2 \\
\hline & & 10.1 & 10.1 & 14.6 & 15.3 \\
\hline \multirow[t]{2}{*}{ Volz et al. 1994a } & \multirow[t]{2}{*}{ TCP } & 73.4 & 80.2 & 1.5 & 1.8 \\
\hline & & 6.3 & 6.0 & 9.0 & 8.5 \\
\hline \multirow[t]{2}{*}{ Volz et al. $1994 \mathrm{~b}$} & \multirow[t]{4}{*}{ TCP } & 54.1 & 60.9 & 8.3 & 6.0 \\
\hline & & 8.1 & 8.4 & 16.0 & 5.3 \\
\hline \multirow[t]{2}{*}{ Total } & & 58.6 & 66.7 & 4.8 & -0.2 \\
\hline & & 6.4 & 7.4 & 5.3 & 17.6 \\
\hline $\begin{array}{l}\text { Number of studies used } \\
\text { in the calculation }\end{array}$ & & [6] & [6] & [6] & {$[6]$} \\
\hline
\end{tabular}

Abbreviations: $\mathrm{IMI}=$ imipramine; $\mathrm{MAP}=$ maprotiline; $\mathrm{LI}=$ lithium; $\mathrm{TCP}=$ tranylcypromine 
Table 4. Summary of Acute Phase Treatment Trials Reporting Categorical Outcomes for Moclobemide (MOC) versus Active Comparators or Placebo in Depressed Outpatients (Mean/Standard Deviation)

\begin{tabular}{|c|c|c|c|c|c|}
\hline \multirow[b]{2}{*}{ Study } & \multirow[b]{2}{*}{ Drug } & \multicolumn{2}{|c|}{ Efficacy } & \multicolumn{2}{|c|}{$\begin{array}{l}\text { Moclobemide } \\
\text { versus Drug/ } \\
\text { Placebo }\end{array}$} \\
\hline & & ITT & AT & ITT & $\mathrm{AT}$ \\
\hline \multirow[t]{2}{*}{ Versiani et al. 1989} & \multirow[t]{2}{*}{ IMI } & 66.9 & 79.5 & 1.8 & 0.4 \\
\hline & & 3.6 & 3.4 & 5.1 & 4.8 \\
\hline \multirow[t]{2}{*}{ Lecrubier and Guelfi 1990} & \multirow{2}{*}{ IMI } & 51.8 & 58.2 & -4.2 & -5.1 \\
\hline & & 3.8 & 4.1 & 5.4 & 5.6 \\
\hline \multirow[t]{2}{*}{ Lecrubier and Guelfi 1990} & \multirow[t]{2}{*}{ CMI } & 80.3 & NA & 19.1 & NA \\
\hline & & 3.9 & & 6.3 & \\
\hline \multirow[t]{2}{*}{ Ucha Udabe et al. 1990} & \multirow[t]{2}{*}{ IMI } & 46.0 & NA & -4.0 & NA \\
\hline & & 9.7 & & 13.8 & \\
\hline \multirow[t]{2}{*}{ Larsen et al. 1991} & \multirow[t]{2}{*}{ CMI } & 45.8 & 65.4 & -13.3 & -23.2 \\
\hline & & 6.3 & 7.2 & 8.9 & 8.7 \\
\hline \multirow[t]{2}{*}{ Larsen et al. 1991} & \multirow[t]{2}{*}{ ISO } & 64.4 & 83.7 & -18.5 & -18.2 \\
\hline & & 6.5 & 5.7 & 9.1 & 9.2 \\
\hline \multirow[t]{2}{*}{ Bakish et al. 1992c } & \multirow[t]{2}{*}{ AMI } & 57.7 & 79.7 & -3.4 & -4.7 \\
\hline & & 6.4 & 6.1 & 9.0 & 8.2 \\
\hline Bougerol et al. 1992 & FLV & 53.7 & 69.6 & 1.3 & -5.9 \\
\hline & & 6.0 & 6.3 & 8.7 & 9.0 \\
\hline Macher and Mirabaud 1992 & AMIN & 53.7 & 93.0 & 22.5 & 34.2 \\
\hline & & 6.0 & 3.1 & 7.2 & 6.4 \\
\hline Steinmeyer et al. 1993 & MAP & 56.7 & 63.2 & 2.4 & 4.8 \\
\hline & & 6.3 & 6.5 & 9.1 & 9.3 \\
\hline Rimon et al. 1993 & IMI & 70.6 & 79.4 & 19.8 & 22.6 \\
\hline & & 5.6 & 3.7 & 8.3 & 8.3 \\
\hline Philipp et al. 1993 & DOX & 41.6 & 52.1 & 86.8 & 8.2 \\
\hline & & 4.4 & 5.0 & 6.2 & 7.2 \\
\hline Beaumont et al. 1993 & DOT & 58.7 & 75.5 & -4.0 & -6.8 \\
\hline & & 3.7 & 3.7 & 5.2 & 4.9 \\
\hline Kusalic et al. 1993 & AMI & 62.5 & NA & -12.5 & NA \\
\hline & & 13.4 & & 17.4 & \\
\hline Gachoud et al. 1994 & MAP & 81.3 & 99.0 & 2.1 & 7.1 \\
\hline & & 4.7 & 1.2 & 6.8 & 3.8 \\
\hline Pancheri et al. 1994 & IMI & 46.8 & 68.1 & -18.7 & 2.5 \\
\hline & & 12.1 & 13.4 & 16.7 & 17.7 \\
\hline Kragh-Sorensen et al. 1995 & CMI & 52.0 & 63.7 & 14.2 & 4.0 \\
\hline & & 7.0 & 7.5 & 9.8 & 11.4 \\
\hline Casacchia et al. 1984 & $\mathrm{PBO}$ & 65.8 & 89.3 & 45.2 & 45.24 \\
\hline & & 10.6 & 8.0 & 14.3 & 5.4 \\
\hline Versiani et al. 1989 & $\mathrm{PBO}$ & 67.0 & 79.5 & 40.3 & 45.24 \\
\hline & & 3.7 & 3.4 & 5.0 & 5.4 \\
\hline Larsen et al. 1989 & $\mathrm{PBO}$ & 28.3 & 38.2 & 4.5 & 6.1 \\
\hline & & 9.2 & 11.5 & 13.2 & 16.6 \\
\hline Lecrubier and Guelfi 1990 & $\mathrm{PBO}$ & 51.8 & 58.2 & 22.7 & 18.9 \\
\hline & & 3.9 & 4.1 & 5.3 & 6.0 \\
\hline Ucha Udabe et al. 1990 & $\mathrm{PBO}$ & 46.0 & NA & 24.0 & NA \\
\hline & & 9.7 & & 12.7 & \\
\hline Botte et al. 1990 & $\mathrm{PBO}$ & 39.6 & NA & 2.6 & NA \\
\hline & & 9.8 & & 12.4 & \\
\hline Bakish et al. 1992c & PBO & 57.8 & 79.8 & 21.1 & 11.4 \\
\hline & & 6.4 & 6.1 & 9.1 & 10.4 \\
\hline Ose \& Holm 1992 & $\mathrm{PBO}$ & 37.5 & 67.5 & 15.4 & 33.4 \\
\hline & & 7.95 & 10.2 & 10.6 & 14.2 \\
\hline Lingjaerde et al. 1993 & $\mathrm{PBO}$ & 44.1 & NA & -0.6 & NA \\
\hline & & 11.7 & & 16.1 & \\
\hline Kusalic et al. 1993 & $\mathrm{PBO}$ & 62.5 & NA & 21.9 & NA \\
\hline & & 13.4 & & 18.0 & \\
\hline
\end{tabular}


Table 4. (continued)

\begin{tabular}{|c|c|c|c|c|c|}
\hline \multirow[b]{2}{*}{ Study } & \multirow[b]{2}{*}{ Drug } & \multicolumn{2}{|c|}{ Efficacy } & \multicolumn{2}{|c|}{$\begin{array}{c}\text { Moclobemide } \\
\text { versus Drug/ } \\
\text { Placebo }\end{array}$} \\
\hline & & ITT & AT & ITT & AT \\
\hline U.K. Study Group 1994 & $\mathrm{PBO}$ & NA & $\begin{array}{r}51.8 \\
6.6\end{array}$ & NA & $\begin{array}{l}3.6 \\
9.4\end{array}$ \\
\hline Nair et al. 1995a & $\mathrm{PBO}$ & $\begin{array}{r}23.0 \\
6.8\end{array}$ & $\begin{array}{l}53.1 \\
12.1\end{array}$ & $\begin{array}{r}10.5 \\
8.7\end{array}$ & $\begin{array}{l}31.7 \\
14.9\end{array}$ \\
\hline Total (Drug) & & $\begin{array}{r}58.9 \\
2.7\end{array}$ & $\begin{array}{r}73.2 \\
3.5\end{array}$ & $\begin{array}{l}2.6 \\
2.5\end{array}$ & $\begin{array}{l}1.7 \\
3.7\end{array}$ \\
\hline Total (PBO) & & $\begin{array}{r}48.7 \\
4.2\end{array}$ & $\begin{array}{r}65.1 \\
4.8\end{array}$ & $\begin{array}{r}15.9 \\
6.2\end{array}$ & $\begin{array}{r}24.2 \\
5.6\end{array}$ \\
\hline $\begin{array}{l}\text { Number of studies used in } \\
\text { the calculation }\end{array}$ & & 27 & 21 & 27 & 21 \\
\hline
\end{tabular}

Abbreviations: $\mathrm{IMI}=$ imipramine; $\mathrm{CMI}=$ clompiramine; $\mathrm{ISO}=$ isocarboxazid; $\mathrm{AMI}=$ amitriptyline; $\mathrm{FLV}=$ fluvoxamine; $\mathrm{AMIN}=$ aminetipine; $\mathrm{MAP}=$ maprotiline; $\mathrm{DOX}=$ doxepin; $\mathrm{DOT}=$ dothiepin; $\mathrm{PBO}=$ placebo.

the ITT samples showed a $2.6 \%(2.5 \%)$ difference (MOC $>$ comparators), and, for the 13 AT samples, the difference in response rates was $1.7 \%(3.7 \%)$. These findings confirm MOC's comparability to other active antidepressants for treatment of ambulatory patient groups.

Inpatient Studies: MOC Versus Active Comparators. A total of five exclusively inpatient RCTs contrasted MOC with other drugs (see Table 4): two with clomipramine (Danish University Antidepressant Group 1993;
Guelfi et al. 1992), and one each versus amitriptyline (Norman et al. 1985), fluoxetine (Prozac) (Gattaz et al. 1995), and fluvoxamine (Luvox) (Bocksberger et al. 1993). Meta-analysis of the five ITT samples yielded a $54.3 \%$ $(8.3 \%)$ MOC response rate. A response rate of $65.2 \%$ $(9.3 \%)$ was observed in the five AT samples. No significant differences were found between MOC and comparators [ITT: $2.7 \%(6.2 \%)$; AT: $0.7 \%(5.2 \%)]$. The evidence from RCTs indicates that MOC is as effective for treatment of more severely depressed inpatients as other antidepressants.

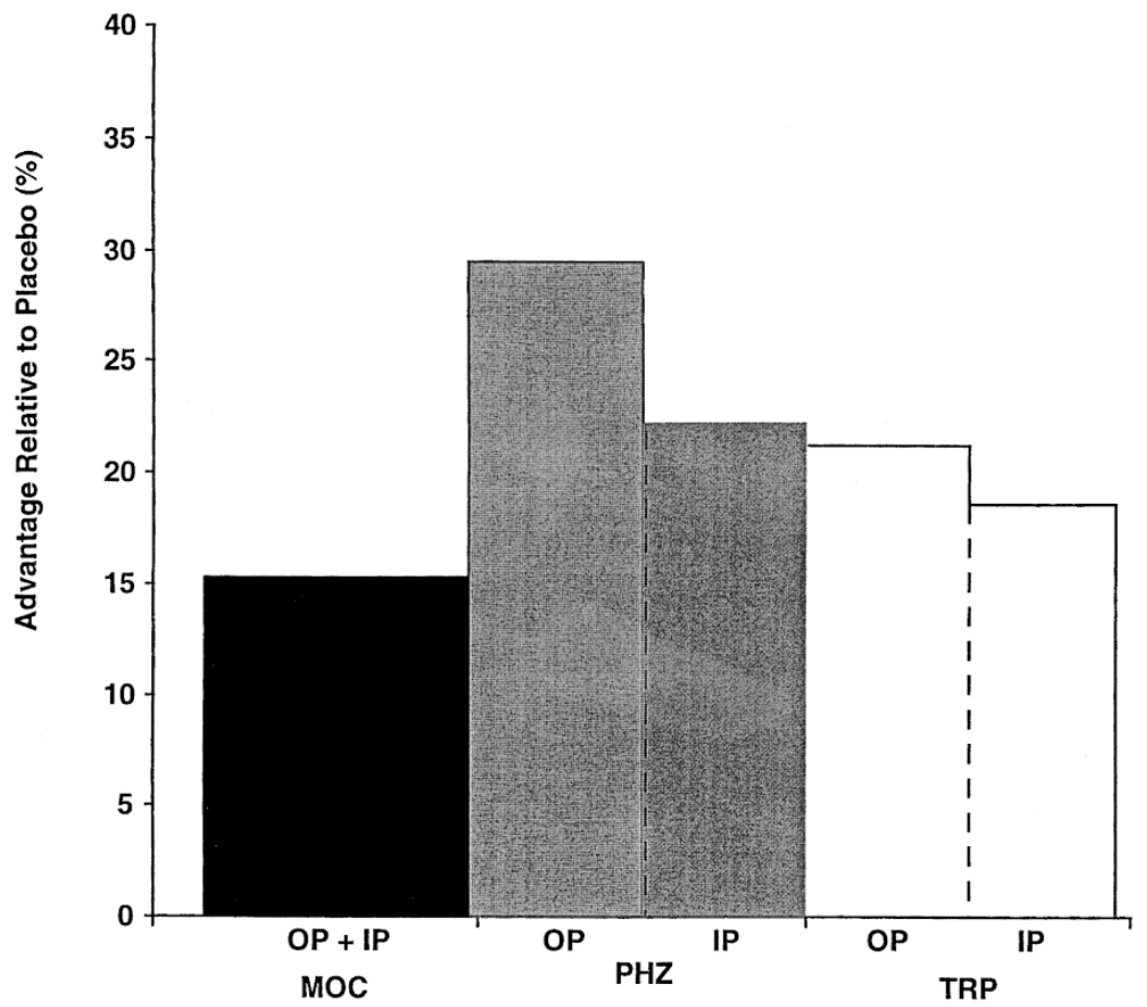

Monoamine Oxidase Inhibitor
Figure 1. Mean drug-placebo differences for moclobemide (MOC), Phenelzine (PHZ), and tranylcypromine (TRP). Note that outpatient (OP) and inpatient (IP) studies are pooled for MOC. Also note that there are inadequate data from inpatient placebo controlled studies of tranylcypromine, so the difference reported is for comparisons of TRP versus active comparators. The actual effect size versus a placebo would undoubtedly be larger. 
Table 5. Acute Phase Treatment Trials Comparing Moclobemide with SSRIs in Depressed Patients (Mean/Standard Deviation)

\begin{tabular}{|c|c|c|c|c|c|}
\hline \multirow[b]{2}{*}{ Study } & \multirow[b]{2}{*}{ Drug } & \multicolumn{2}{|c|}{ Efficacy } & \multicolumn{2}{|c|}{$\begin{array}{l}\text { Moclobemide } \\
\text { versus SSRIs }\end{array}$} \\
\hline & & ITT & AT & ITT & AT \\
\hline \multirow[t]{2}{*}{ Bougerol et al. 1992} & \multirow[t]{2}{*}{ FLV } & 53.8 & 69.6 & 1.4 & -5.97 \\
\hline & & 6.1 & 6.4 & 8.8 & 9.09 \\
\hline \multirow[t]{2}{*}{ Bocksberger et al. 1993} & \multirow[t]{2}{*}{ FLV } & 78.6 & 82.5 & 23.8 & 21.9 \\
\hline & & 8.7 & 8.3 & 13.7 & 13.7 \\
\hline \multirow[t]{2}{*}{ Williams et al. 1993} & \multirow[t]{2}{*}{ FLU } & 61.1 & 61.1 & 7.8 & 9.5 \\
\hline & & 6.1 & 6.1 & 8.8 & 8.74 \\
\hline \multirow[t]{2}{*}{ Lonnqvist et al. 1994b } & \multirow[t]{2}{*}{ FLU } & 66.5 & 80.5 & 9.5 & 6.9 \\
\hline & & 4.6 & 4.3 & 6.6 & 6.45 \\
\hline \multirow[t]{2}{*}{ Geertz et al. 1995} & \multirow[t]{2}{*}{ FLU } & 50.0 & 50.0 & 13.4 & 10.2 \\
\hline & & 9.8 & 9.8 & 3.5 & 15.68 \\
\hline \multirow[t]{2}{*}{ Reynaert et al. 1995} & \multirow[t]{2}{*}{ FLU } & 37.5 & 50.0 & -4.6 & 0 \\
\hline & & 6.7 & 7.9 & 4.4 & 10.92 \\
\hline \multirow[t]{2}{*}{ Gattaz et al. 1995} & \multirow[t]{2}{*}{ FLU } & 58.1 & 76.7 & -0.5 & 0.85 \\
\hline & & 8.0 & 7.8 & 11.5 & 11.26 \\
\hline \multirow[t]{2}{*}{ Total } & & 58.1 & 67.9 & 6.5 & 4.87 \\
\hline & & 3.4 & 3.8 & 4.0 & 4.12 \\
\hline $\begin{array}{l}\text { Number of studies } \\
\text { used in the calculation }\end{array}$ & & 8 & 8 & 8 & 8 \\
\hline
\end{tabular}

Abbreviations: FLV = fluvoxamine, FLU = fluoxetine.

Moclobemide versus SSRIs. Across inpatient and outpatient settings, eight studies compared MOC with SSRIs. There were two contrasts with fluvoxamine and six against fluoxetine (see Table 5). In these eight trials, MOC had an ITT response rate of $58.0 \%(3.4 \%)$. In the AT samples, the MOC response rate was $67.9 \%$ (3.8\%). The difference between MOC and the SSRIs was $6.4 \%$ $(3.9 \%)$ in the ITT analysis, and, for AT sample, the difference was of $4.8 \%(4.1 \%)$. In both cases, there was a small but reliable advantage favoring MOC over the SSRIs. However, the clinical significance of these such small differences in response rates is certainly debatable.

Moclobemide Contrasted with Older MAOIs. Four studies were available (one with isocarboxazid and three with tranylcypromine) for the ITT comparison, although just two were suitable for the AT comparison (see Table 6). The overall ITT efficacy of MOC was $68.0 \%(7.3 \%)$ and the AT efficacy was $71.6 \%(6.8 \%)$. The ITT difference was $-5.8 \%(8.4 \%)$, and the AT differences was $-13.3 \%(6.8 \%)$ in favor of the nonselective MAOIs. Furthermore, in the one trial in which MOC was superior to tranylcypromine (Gabelic and Kuhn 1990), the dosage of the older MAOI drug was clearly subtherapeutic (i.e., $10-30 \mathrm{mg} /$ day).

Moclobemide Contrasted with Tricyclics. Across inpatient and outpatients studies, 29 reports compared MOC with a TCA (see Table 7). The comparators were clomipramine $(n=10)$, imipramine $(n=9)$, amitriptyline $(n=5)$, doxepin $(n=2)$, and desipramine, nortriptyline and dothiepin (one study each). Twenty- eight studies were suitable for the ITT analysis and 23 for the AT analysis. The ITT response rate for MOC was $54.6 \%(2.9 \%)$, and the AT response rate was $63.6 \%$ (3.1\%). The ITT difference between MOC and the TCAs was $1.8 \%(2.3 \%)$. The AT difference was $-1.2 \%(2.0 \%)$ (TCA $>$ MOC). See Table 8 and Table 9.

Moclobemide Studies of Elderly Samples. Five reports were identified that exclusively enrolled elderly patients (see Table 10). Comparators included imipramine

Table 6. Acute Phase Treatment Trials Comparing Moclobemide with a Nonselective MAOI

(Mean/Standard Deviation)

\begin{tabular}{|c|c|c|c|c|c|}
\hline \multirow[b]{2}{*}{ Study } & \multirow[b]{2}{*}{ Drug* } & \multicolumn{2}{|c|}{ Efficacy } & \multicolumn{2}{|c|}{$\begin{array}{l}\text { Brofaromine } \\
\text { Versus Drug }\end{array}$} \\
\hline & & ITT & AT & ITT & AT \\
\hline Gabelic and Kuhn 1990 & TCP & $\begin{array}{r}88.1 \\
6.9\end{array}$ & NA & $\begin{array}{l}28.5 \\
12.5\end{array}$ & NA \\
\hline Rossel and Moll 1990 & TCP & $\begin{array}{r}69.1 \\
9.9\end{array}$ & NA & $\begin{array}{r}-14.3 \\
12.7\end{array}$ & NA \\
\hline Larsen et al. 1991 & ISO & $\begin{array}{r}45.8 \\
6.4\end{array}$ & $\begin{array}{r}65.5 \\
7.3\end{array}$ & $\begin{array}{r}-18.6 \\
9.2\end{array}$ & $\begin{array}{r}-18.3 \\
9.3\end{array}$ \\
\hline Heinze et al. 1993 & ТCP & $\begin{array}{r}76.2 \\
4.7\end{array}$ & $\begin{array}{r}77.2 \\
4.6\end{array}$ & $\begin{array}{r}-9.4 \\
6.1\end{array}$ & $\begin{array}{r}-10.7 \\
5.9\end{array}$ \\
\hline Total & & $\begin{array}{r}68.1 \\
7.4\end{array}$ & $\begin{array}{r}71.7 \\
6.9\end{array}$ & $\begin{array}{r}-5.8 \\
8.5\end{array}$ & $\begin{array}{r}-13.4 \\
6.8\end{array}$ \\
\hline $\begin{array}{l}\text { Number of studies used } \\
\text { in the calculation }\end{array}$ & & 4 & 2 & 4 & 2 \\
\hline
\end{tabular}

${ }^{*} \mathrm{TCP}=$ tranylcypromine; $\mathrm{ISO}=$ isocarboxazide. 
Table 7. Acute Phase Treatment Trials Comparing Moclobemide with TCAs (Mean/Standard Deviation)

\begin{tabular}{|c|c|c|c|c|c|}
\hline \multirow[b]{2}{*}{ Study } & \multirow[b]{2}{*}{ Drug } & \multicolumn{2}{|c|}{ Efficacy } & \multicolumn{2}{|c|}{$\begin{array}{l}\text { Moclobemide } \\
\text { Versus TCA2 }\end{array}$} \\
\hline & & ITT & AT & ITT & AT \\
\hline \multirow[t]{2}{*}{ Norman et al. 1985} & \multirow[t]{2}{*}{ AMI } & 60.7 & 60.7 & 3.0 & 3.0 \\
\hline & & 12.6 & 12.6 & 18.2 & 18.2 \\
\hline \multirow[t]{2}{*}{ Koczkas et al. 1989} & \multirow[t]{2}{*}{ CMI } & 31.8 & 52.5 & -5.2 & $0 ., 2$ \\
\hline & & 7.9 & 10.9 & 11.6 & 15.0 \\
\hline \multirow[t]{2}{*}{ Versiani et al. 1989} & \multirow[t]{2}{*}{ IMI } & 66.9 & 79.5 & 1.8 & 0.4 \\
\hline & & 3.6 & 3.4 & 5.1 & 4.8 \\
\hline \multirow[t]{2}{*}{ Casacchia and Rossi 1989} & \multirow[t]{2}{*}{ IMI } & 73.8 & 91.1 & 9.5 & 6.8 \\
\hline & & 9.3 & 6.6 & 13.8 & 11.0 \\
\hline \multirow[t]{2}{*}{ Baumhackl et al. 1989} & \multirow[t]{2}{*}{ IMI } & 59.7 & 73.2 & 4.0 & 10.7 \\
\hline & & 3.5 & 3.5 & 5.0 & 5.1 \\
\hline \multirow[t]{2}{*}{ Larsen et al. 1989} & \multirow[t]{2}{*}{ CMI } & 28.2 & 38.2 & -26.5 & -25.6 \\
\hline & & 9.1 & 11.4 & 14.4 & 15.8 \\
\hline \multirow[t]{2}{*}{ Casacchia and Moll 1990} & \multirow[t]{2}{*}{ IMI } & 78.5 & NA & 23.8 & NA \\
\hline & & 8.7 & & 13.7 & \\
\hline \multirow[t]{2}{*}{ Beckers et al. 1990} & \multirow[t]{2}{*}{ AMI } & 83.3 & NA & 38.3 & NA \\
\hline & & 11.7 & & 19.0 & \\
\hline Beckers et al. 1990 & AMI & 67.8 & NA & 11.1 & NA \\
\hline & & 12.0 & & 17.2 & \\
\hline Dierick et al. 1990 & CMI & 37.8 & 41.6 & 5.0 & -0.3 \\
\hline & & 8.3 & 8.8 & 11.6 & 13.1 \\
\hline Civeira et al. 1990 & CMI & 54.4 & 66.0 & -9.6 & -9.8 \\
\hline & & 8.4 & 8.7 & 11.8 & 11.9 \\
\hline Gabelic and Moll 1990 & DMI & 78.1 & 83.3 & 43.7 & 28.3 \\
\hline & & 10.0 & 9.3 & 15.2 & 17.6 \\
\hline Lecrubier and Guelfi 1990 & IMI & 51.8 & 51.8 & -4.2 & -5.1 \\
\hline & & 3.8 & 4.0 & 5.4 & 5.6 \\
\hline Lecrubier and Guelfi 1990 & CMI & 80.3 & NA & 19.1 & NA \\
\hline & & 3.9 & & 6.3 & \\
\hline Ucha Udabe et al. 1990 & IMI & 46.0 & NA & -4.0 & NA \\
\hline & & 9.7 & & 13.8 & \\
\hline Funke et al. 1990 & CMI & 46.8 & 50.0 & -6.2 & -3.1 \\
\hline & & 12.2 & 12.5 & 17.1 & 17.4 \\
\hline Larsen et al. 1991 & CMI & 45.8 & 65.4 & -15.3 & -23.2 \\
\hline & & 6.3 & 7.2 & 87.9 & 8.7 \\
\hline Guelfi et al. 1992 & CMI & 64.2 & 84.3 & -7.0 & -0.7 \\
\hline & & 5.9 & 5.1 & 8.0 & 6.9 \\
\hline Bakish et al. 1992c & AMI & 57.7 & 79.7 & 21.1 & -4.7 \\
\hline & & 6.4 & 6.1 & 9.0 & 6.9 \\
\hline Danish UAG 1993 & CMI & 19.8 & 22.1 & -13.2 & -12.1 \\
\hline & & 5.1 & 5.7 & 7.9 & 8.4 \\
\hline Rimon et al. 1993 & IMI & 70.6 & 79.4 & 19.8 & 22.6 \\
\hline & & 5.6 & 5.3 & 8.3 & 8.3 \\
\hline Philipp et al. 1993 & DOX & 41.6 & 52.1 & 8.6 & 8.2 \\
\hline & & 4.4 & 5.0 & 6.2 & 7.2 \\
\hline Beaumont et al. 1993 & DOT & 58.7 & 75.5 & -4.0 & -6.8 \\
\hline & & 3.7 & 3.7 & 5.2 & 4.9 \\
\hline Kusalic et al. 1993 & AMI & 62.5 & NA & -12.5 & NA \\
\hline & & 13.4 & & 17.4 & \\
\hline U.K.S.G. 1994 & IMI & NA & 51.7 & NA & -6.0 \\
\hline & & 6.5 & & 9.4 & \\
\hline
\end{tabular}

(continued)

(Pancheri et al. 1994), mianserin (De Vanna et al. 1990; Tiller et al. 1990), fluvoxamine (Bocksberger et al. 1993), and nortriptyline (Aventyl) (Nair et al. 1995b). The ITT response rate was $47.8 \%(8.0 \%)$, and the AT response
Table 7. (continued)

\begin{tabular}{|c|c|c|c|c|c|}
\hline \multirow[b]{2}{*}{ Study } & \multirow[b]{2}{*}{ Drug* } & \multicolumn{2}{|c|}{ Efficacy } & \multicolumn{2}{|c|}{$\begin{array}{l}\text { Moclobemide } \\
\text { versus TCAs }\end{array}$} \\
\hline & & ITT & AT & ITT & AT \\
\hline \multirow[t]{2}{*}{ Pancheri et al. 1994} & \multirow[t]{2}{*}{ IMI } & 46.8 & 53.1 & -18.7 & 2.5 \\
\hline & & 12.1 & 12.1 & 16.7 & 17.7 \\
\hline \multirow[t]{2}{*}{ Nair et al. 1995a } & \multirow[t]{2}{*}{ NOR } & 22.9 & 53.1 & -9.0 & -6.3 \\
\hline & & 6.8 & 12.1 & 10.0 & 16.0 \\
\hline \multirow[t]{2}{*}{ Kragh-Sorensen et al. 1995} & \multirow[t]{2}{*}{ CMI } & 52.0 & 63.7 & 14.2 & 4.0 \\
\hline & & 7.0 & 7.5 & 9.8 & 11.4 \\
\hline \multirow[t]{2}{*}{ Lingjaerde et al. 1995} & \multirow[t]{4}{*}{ DOX } & 59.6 & 68.5 & -17.4 & -19.5 \\
\hline & & 8.6 & 8.7 & 12.0 & 11.1 \\
\hline \multirow[t]{2}{*}{ Total } & & 54.6 & 63.6 & 1.8 & -1.2 \\
\hline & & 2.9 & 3.1 & 2.3 & 2.0 \\
\hline \multicolumn{2}{|l|}{$\begin{array}{l}\text { Number of studies used } \\
\text { in calculation }\end{array}$} & 28 & 23 & 28 & 23 \\
\hline
\end{tabular}

Abbreviations: $\mathrm{AMI}=$ amitriptyline, $\mathrm{CMI}=$ clomipramine, $\mathrm{IMI}=$ imipramine, $\mathrm{DMI}=$ desipraine, $\mathrm{DOX}=$ doxepin, $\mathrm{NOR}=$ nortriptyline

rate was $69.9 \%(6.4 \%)$. In the ITT sample, the difference between MOC and the comparators were $1.3 \%(7.6 \%)$, and, in the AT sample, it was $6.3 \%(8.4 \%)$.

Safety During Acute Phase Therapy. Moll and Hetzel (1990) compared side effects in 2203 patients who were treated with MOC and 1214 patients treated with other antidepressants. The most common adverse events during MOC therapy involved the central nervous system (including insomnia, 20.5\%), followed by gastrointestinal symptoms such as diarrhea and nausea (19.9\%). There were no significant differences between moclobemide and placebo for any of the serious adverse events. Except for increased incidence of nausea and insomnia, MOC was generally better tolerated than tricyclics (Moll and Hetzel, 1990). In a separate report covering a largely overlapping database, only one hypertensive crisis was reported during MOC therapy (Versiani et al. 1990). This would suggest an incidence of less than $0.1 \%$.

Chen and Ruch (1993) examined the safety of MOC therapy in a newer and, apparently, independent database. There were 1291 moclobemide-treated patients, compared to 810 placebo-treated patients. The most frequent and (relative to $\mathrm{PBO}$ ) significantly greater reported adverse events were dizziness, nausea, and insomnia. When compared to 1288 patients treated with TCAs, MOC therapy was associated with significantly lower incidence of dry mouth, sweating, tremor, somnolence, dizziness, blurred vision, and sexual side effects. However, MOC therapy resulted in significantly more complaints of headaches and insomnia than the TCAs.

Continuation/Maintenance Treatment. Stefanis and Merz-Frei (1990) followed-up 102 MOC responders for 1 year of prophylactic treatment. Efficacy was judged to be good or very good for $96 \%$ of patients. An assess- 
Table 8. Acute Phase Treatment Trials Comparing Moclobemide with Other (non TCA, non SSRI) Antidepressants (Mean/Standard Deviation)

\begin{tabular}{|c|c|c|c|c|c|}
\hline \multirow[b]{2}{*}{ Study } & \multirow[b]{2}{*}{ Drug } & \multicolumn{2}{|c|}{ Efficacy } & \multicolumn{2}{|c|}{$\begin{array}{l}\text { Moclobemide } \\
\text { versus Drug }\end{array}$} \\
\hline & & ITT & AT & ITT & AT \\
\hline \multirow[t]{2}{*}{ De Vanna et al. 1990} & \multirow[t]{2}{*}{ MIA } & 59.8 & 74.2 & 0 & 4.2 \\
\hline & & 7.6 & 7.5 & 10.7 & 10.7 \\
\hline \multirow[t]{2}{*}{ Classen and Laux 1990} & \multirow[t]{2}{*}{ MAP } & 40.5 & 60.7 & -9.5 & 5.5 \\
\hline & & 10.4 & 12.2 & 14.9 & 16.8 \\
\hline \multirow[t]{2}{*}{ Tiller 1990} & \multirow[t]{2}{*}{ MIA } & 32.1 & NA & 14.3 & NA \\
\hline & & 12.1 & & 15.6 & \\
\hline \multirow[t]{2}{*}{ Macher and Mirabaud 1992} & \multirow[t]{2}{*}{ AMIN } & 75.6 & 93.1 & 22.6 & 34.3 \\
\hline & & 4.8 & 3.1 & 7.3 & 6.5 \\
\hline \multirow[t]{2}{*}{ Steinmeyer et al. 1993} & \multirow[t]{2}{*}{ MAP } & 56.8 & 63.2 & 2.5 & 4.9 \\
\hline & & 6.4 & 6.6 & 9.1 & 9.3 \\
\hline \multirow[t]{2}{*}{ Vaz Serra et al. 1994} & \multirow[t]{2}{*}{ MAP } & 81.7 & 85.9 & 36.6 & 37.2 \\
\hline & & 6.0 & 5.5 & 9.7 & 9.7 \\
\hline \multirow[t]{2}{*}{ Gachoud et al. 1994} & \multirow[t]{2}{*}{ MAP } & 81.3 & 99.1 & 2.1 & 7.1 \\
\hline & & 4.7 & 1.3 & 6.9 & 3.8 \\
\hline \multirow[t]{2}{*}{ Total } & & 64.7 & 80.5 & 10.6 & 16.1 \\
\hline & & 5.6 & 5.8 & 5.0 & 5.8 \\
\hline $\begin{array}{l}\text { Number of studies } \\
\text { used in the calculation }\end{array}$ & & 7 & 6 & 7 & 6 \\
\hline
\end{tabular}

Abbreviations: $\mathrm{MIA}=$ mianserin, $\mathrm{MAP}=$ maprotiline, $\mathrm{AMIN}=$ amineptine

ment combining efficacy and tolerability was rated as good or very good for $85 \%$ of patients.

Gagiano et al. (1994) reported on a 6-month, open-label study of MOC continuation therapy (300-450 mg/day). Just 6 of 81 patients $(7.5 \%)$ relapsed, although another 10 patients $(12 \%)$ were lost to follow-up. Thus, sustained efficacy across 6 months of continuation therapy was at least $80.5 \%$. Among those who completed fol-

Table 9. Summary of Acute Phase Treatment Trials Reporting Categorical Outcomes for Moclobemide (MOC) in Depressed Inpatients (Mean/Standard Deviation)

\begin{tabular}{|c|c|c|c|c|c|}
\hline \multirow[b]{2}{*}{ Study } & \multirow[b]{2}{*}{ Drug } & \multicolumn{2}{|c|}{$\begin{array}{l}\text { Overall } \\
\text { Efficacy }\end{array}$} & \multicolumn{2}{|c|}{$\begin{array}{l}\text { Relative } \\
\text { Efficacy }\end{array}$} \\
\hline & & ITT & AT & ITT & AT \\
\hline \multirow[t]{2}{*}{ Norman et al. 1985} & \multirow[t]{2}{*}{ AMI } & 60.7 & 60.7 & 3.0 & 3.0 \\
\hline & & 12.6 & 12.6 & 18.2 & 18.2 \\
\hline \multirow[t]{2}{*}{ Guelfi et al. 1992} & \multirow[t]{2}{*}{ CMI } & 64.2 & 84.3 & -7.0 & -0.7 \\
\hline & & 5.9 & 5.1 & 8.1 & 6.9 \\
\hline \multirow[t]{2}{*}{ Danish UAG 1993} & \multirow[t]{2}{*}{$\mathrm{CMI}$} & 19.8 & 22.1 & -13.2 & -12.1 \\
\hline & & 5.1 & 5.7 & 7.9 & 8.4 \\
\hline \multirow[t]{2}{*}{ Bocksberger et al. 1993} & \multirow[t]{2}{*}{ FLV } & 78.5 & 82.5 & 23.8 & 21.9 \\
\hline & & 8.7 & 8.3 & 13.8 & 13.7 \\
\hline \multirow[t]{2}{*}{ Gattaz et al. 1995} & \multirow[t]{2}{*}{ FLU } & 58.1 & 76.7 & -0.5 & 0.89 \\
\hline & & 8.0 & 7.8 & 11.4 & 11.2 \\
\hline \multirow[t]{2}{*}{ Total } & & 54.4 & 65.3 & -2.7 & -0.7 \\
\hline & & 8.3 & 9.3 & 6.2 & 5.2 \\
\hline $\begin{array}{l}\text { Number of studies used } \\
\text { in the calculation }\end{array}$ & & 5 & 5 & 5 & 5 \\
\hline
\end{tabular}

Abbreviations: $\mathrm{PBO}=$ placebo, $\mathrm{AMI}=$ amitriptyline, $\mathrm{CMI}=$ clomipramine, $F L V=$ fluvoxamine, FLU = fluoxetine. low-up, there was a further $40 \%$ reduction in Hamilton scores as compared to the end of acute phase therapy. No patient who completed the study developed serious side effects.

Lonnqvist et al. (1995) studied continuation therapy in outpatients who responded to acute phase therapy with either MOC $(n=29)$ or fluoxetine $(n=30)$. Assessments were conducted without knowledge of the treatment for 12 additional weeks. Only 3 of the 59 patients (5\%) relapsed during continuation therapy (two patients in the fluoxetine group and one in the MOC group). The number of drop-outs did not differ significantly, and adverse event complaints decreased by $67 \%$ during continuation phase treatment.

Across these three studies, continuation therapy with MOC was associated with sustained response rates that would have definitely surpassed those observed in PBO-controlled discontinuation designs (see, for example, the review by Thase and Sullivan, 1995). Although prophylaxis against recurrent depression has not been established empirically, the favorable tolerability of MOC observed during continuation phase therapy is promising when compared to longer-term studies of the older MAOIs (e.g., Robinson et al. 1991; Stewart et al. 1997).

\section{CONCLUSIONS}

The results of these meta-analyses indicate that both $\mathrm{BRO}$ and MOC are effective antidepressants for both in- 
Table 10. Acute Phase Treatment Trials Comparing Moclobemide with Other Antidepressants in Depressed Geriatric Patients (Mean/Standard Deviation)

\begin{tabular}{|c|c|c|c|c|c|}
\hline \multirow[b]{2}{*}{ Study } & \multirow[b]{2}{*}{ Drug } & \multicolumn{2}{|c|}{ Efficacy } & \multicolumn{2}{|c|}{$\begin{array}{l}\text { Moclobemide } \\
\text { versus Drug }\end{array}$} \\
\hline & & ITT & AT & ITT & AT \\
\hline \multirow[t]{2}{*}{ DeVanna et al. 1990} & \multirow[t]{2}{*}{ MIA } & 59.7 & 74.2 & 0 & 4.2 \\
\hline & & 7.5 & 7.5 & 10.7 & 10.7 \\
\hline \multirow[t]{2}{*}{ Tiller et al. 1990} & \multirow[t]{2}{*}{ MIA } & 32.1 & NA & 14.2 & NA \\
\hline & & 12.0 & & 15.5 & \\
\hline \multirow[t]{2}{*}{ Bocksberger et al. 1993} & \multirow[t]{2}{*}{ FLV } & 78.57 & 82.5 & 23.81 & 21.97 \\
\hline & & 8.7 & 8.29 & 13.75 & 13.72 \\
\hline \multirow[t]{2}{*}{ Pancheri et al. 1994} & \multirow[t]{2}{*}{ IMI } & 46.8 & 68.1 & -18.7 & 2.5 \\
\hline & & 12.1 & 13.4 & 16.7 & 17.7 \\
\hline \multirow[t]{2}{*}{ Nair et al. 1995b } & \multirow[t]{2}{*}{ NOR } & 22.9 & 53.1 & -9.0 & -6.3 \\
\hline & & 6.8 & 12.1 & 10.0 & 16.0 \\
\hline \multirow[t]{2}{*}{ Total } & & 47.8 & 69.9 & 1.35 & 6.25 \\
\hline & & 8.02 & 6.4 & 7.6 & 8.4 \\
\hline $\begin{array}{l}\text { Number of studies } \\
\text { used in the calculation }\end{array}$ & & 5 & 4 & 5 & 4 \\
\hline
\end{tabular}

Abbreviations: $\mathrm{MIA}=$ mianserin, $\mathrm{FLV}=$ fluvoxamine, $\mathrm{IMI}=$ imipramine, $\mathrm{NOR}=$ nortriptyline

patients and outpatients. Although there are fewer data, MOC appears to be at least as effective as the SSRIs, if not more so. Specifically, a $6.4 \%(\mathrm{SD}=+3.9 \%)$ difference favoring MOC over SSRIs was found in the ITT samples of eight published studies. Although BRO may be inferred to be as effective as MOC on the basis of studies using active comparators, there are no "headto-head" comparisons, and only MOC has been studied adequately using $\mathrm{PBO}$ control groups.

Both MOC and BRO appear to be safer and better tolerated than the older, nonselective, and irreversible MAOIs. Tolerability also appears to favor the RIMAs when compared to TCAs, although these differences were less pronounced. In all, the safety and tolerability advantage of the RIMAs appears to be similar to that of the SSRIs. Preliminary evidence also suggests that MOC has good longer-term tolerability and sustained prophylactic efficacy.

Although the experience prescribing is limited in RIMAs in the United States, many clinicians in Mexico, Canada, Brazil, the United Kingdom, and Europe believe that MOC is not as effective as nonselective MAOIs such as tranylcypromine or phenelzine. Consistent with these clinical impressions, the results of our meta-analysis indicate a clinically significant advantage for the older MAOIs [13.3\% (6.8\%)] in the "adequate trial" comparison. It should be noted, however, that this conclusion is based on only four direct comparisons and is hardly definitive.

If true, what could account for the difference between MOC and tranylcypromine or phenelzine? On one hand, the inhibition of both the $\mathrm{A}$ and $\mathrm{B}$ MAO isoenzymes may convey a stronger antidepressant effect by enhancing dopaminergic neurotransmission or by increasing levels of trace biogenic amines such as phenylethylamine. Reversibility of MAO inhibition, in and of itself, also may be associated with less robust or pronounced effects on central monoaminergic systems through some as yet unexplained mechanism. It is also possible that the optimal dose of MOC for treatment of more severe or refractory mood disorders is higher than currently appreciated. For example, Angst et al. (1995) have marshalled data to suggest that MOC at doses of $\leqslant 400 \mathrm{mg} /$ day is a less effective treatment of severely depressed inpatients than either clomipramine $(\geqslant 150$ $\mathrm{mg} /$ day) or MOC at higher dosages ( $\geqslant 450 \mathrm{mg} /$ day). It would appear, then, that prescription of larger doses (i.e., 450 to $900 \mathrm{mg}$ /day) would be a rational first step for management of an ineffective but well-tolerated trial of MOC.

BRO is currently not approved for general use anywhere in the world, and, as a result, there is less anecdotal evidence about its relative merits in comparison to the older MAOIs. In contrast to the analyses of MOC, the three studies that contrasted BRO with tranylcypromine failed to yield any evidence of an advantage for the older MAOI (Nolen et al. 1993; Volz et al 1994a, b). These findings are consistent with the possibility that the 5-HT reuptake inhibitory effects of BRO conveys a stronger therapeutic effect than moclobemide. Alternatively, perhaps the fact that BRO is a relatively less reversible inhibitor of MAO Type A than MOC is important. Unfortunately, we were not able to identify any reports examining response to $\mathrm{BRO}$ after unsuccessful treatment with therapeutic doses of MOC.

Of course, too much emphasis should not be placed on indirect comparisons across meta-analyses. Moreover, the results of the three studies that compared BRO and tranylcypromine can also be questioned because none used what could be considered to be standard 
doses of tranylcypromine (Thase et al. 1995). Specifically, the two studies reported by Volz and associates (1994a, b) employed doses of tranylcypromine (20 to 30 $\mathrm{mg}$ /day) that we would consider to be subtherapeutic, whereas Nolen et al. (1993) prescribed an uncharacteristically large mean daily dosage $(84 \mathrm{mg} /$ day). This issue certainly warrants further study if BRO "resurfaces" from pharmacologic limbo.

Based on these meta-analyses, we conclude that, there is little reason to favor the RIMAs over the SSRIs. Although the RIMAs might have a slight advantage with respect to efficacy, the SSRIs are somewhat more convenient to prescribe (e.g., single daily dosing and, possibly, less dosage titration), and there is a much more extensive track record of safety, especially in overdose. With respect to side effects, the RIMAs have only one clear advantage over the SSRIs, namely a lower incidence of sexual dysfunction. Available evidence suggests that patients treated with RIMAs may experience more insomnia than those taking SSRIs, although this side effect is problematic for both classes.

It is not clear to what extent the characteristics of patients who respond to SSRIs and RIMAs overlap. This has important implications for decisions about sequential treatment strategies for patients who do not respond to first-line antidepressants. Like the older MAOIs and SSRIs, MOC and BRO have shown promise for treatment of panic and other anxiety states (Bakish 1992a; Van Vliet et al. 1993). However, the RIMAs have not yet established a strong track record as a preferred treatment of depressive episodes characterized by reverse vegetative features (Larsen et al. 1991; Lonnqvist et al. 1994a). Similarly, it is not clear that MOC shares the potential advantages of tranylcypromine (relative to the TCAs) for treatment of anergic bipolar depression (Himmelhoch et al. 1991; Thase et al. 1992a) or TCAresistant recurrent unipolar depressions (Thase et al. 1992b). For example, in a report using a pooled data set, Angst and Stabl (1992) found that MOC and TCAs had comparable response rates in bipolar depression.

The limitations of meta-analysis also should be acknowledged. The results of a meta-analysis are influenced by the quality of the individual studies being evaluated and are subject to systematic bases. One particular problem is the generalizability of data from controlled clinical trials, especially when most patients with severe medical illnesses or marked psychiatric comorbidity have been excluded.

Finally, we note that neither MOC nor BRO will be available in the United States at any point in the foreseeable future. Although our meta-analysis indicates that these drugs are effective and safe antidepressants, there is apparently insufficient financial incentive to support the additional research that would be necessary to receive approval from the Food and Drug Administration. Moreover, this decision was apparently made for BRO worldwide (Volz et al. 1995). Thus, for psychiatrists practicing in the United States, BRO and MOC are functionally orphan drugs, even though the conditions they treat are ubiquitous and the older MAOIs leave much to be desired. Based on the sales of other novel antidepressants recently introduced in the United States (e.g., venlafaxine [Effexor], nefazodone [Serzone], and mirtazapine [Remeron]), we suspect that the RIMAs would "capture" about $1 \%$ or $2 \%$ of the U.S. market share of name-brand antidepressants, which would limit potential profitability. Nevertheless, even such modest sales would offset the costs of necessary Phase III studies, and these drugs would represent a useful addition to our therapeutic arsenal. Patients who cannot tolerate the older MAOIs or who do not respond to SSRIs are the most likely to benefit if these compounds were available. Can't a good home in the United States be found for these orphans?

\section{ACKNOWLEDGMENTS}

This work was supported in part by grant MH-30915 (Mental Health Clinical Research Center). The authors wish to thank Christine Johnson and Lisa Stupar for their help in completing the manuscript.

\section{REFERENCES}

Allain H, Lieury A, Brunet-Bourgin F, Mirabaud C, Trebon P, Le Coz F, Gandon JM (1992): Antidepressants and cognition: comparative effects of moclobemide, viloxazine and maprotiline. Psychopharmacol 106:S56-S61

Angst J, Amrein R, Stabl M (1995): Moclobemide and tricyclic antidepressants in severe depression: meta-analysis and prospective studies. J Clin Psychopharmacol 4(Suppl 2):16S-23S

Angst J, Scheidegger P, Stabl M (1993): Efficacy of moclobemide in different patient groups. Results of new subscales of the Hamilton Depression Rating Scale. Clin Neuropharmacol 16(Suppl 2):S55-S62

Angst J, Stabl M (1992): Efficacy of moclobemide in different patient groups: a meta-analysis of studies. Psychopharmacol 106(Suppl):S109-S113

Bakish D (1992a): Reversible monoamine-A inhibitors in panic disorder. Clin Neuropharmacol 15:432A-433A

Bakish D, Bradwejn J, Nair N, McClure J, Remick R, Bulger L (1992b): A comparison of moclobemide, amitriptyline and placebo in depression: a Canadian multicentre study. Psychopharmacol 106:S98-S101

Bakish D, Wiens A, Ellis J, Alda M, LaPierre Y (1992c): A double-blind placebo-controlled comparison of moclobemide and amitriptyline in the treatment of depression. Can J Psychiatry 37(Suppl 1):12-17

Barrelet L, Blajev B, Bolzani L, de Saussure C, Kasas A, Van H, Gachoud JP (1991): Multicenter study comparing efficacy and tolerance of moclobemide and fluvoxamine in hospitalized and ambulatory patients with severe 
depressive episodes. Schweiz Rundschaw Med Praxis 80(19):524- 528

Baumhackl U, Biziere K, Fischbach R, Geretsegger Ch, Hebenstreit G, Radmayr E, Stabl M (1989): Efficacy and tolerability of moclobemide compared with imipramine in depressive disorder (DSM- III): an Austrian doubleblind, multicentre study. Br J Psychiatry 155(Suppl 6):78-83

Beasley CM, Masica DN, Heiligenstein JH, Wheadon DE, Zerbe RL (1993): Possible monoamine oxidase inhibitorserotonin uptake inhibitor interaction: fluoxetine clinical data and preclinical findings. J Clin Psychopharmacol 13(5):312-320

Beaumont G, Gringras M, Hobbs FDR, Drury VWM, Freeling P, Tylee A, Shanley DF, Comber H, Sittle S, Hill S (1993): A randomized, double-blind, multi-centre, parallelgroup study comparing the tolerability and efficacy of moclobemide and dothiepin hydrochloride in depressed patients in general practice. Int Clin Pharmacol 7:159_ 165

Beckers G, Vereecken A, de Bleeker E, Jaunes C, Sieben G, Faidherbe J, Wolfrum C, Berger M, Hellstern K, Ward J (1990): Moclobemide versus amitriptyline in the treatment of depression: two small double-blind multicentre studies in Belgium. Acta Psychiatr Scand 82(Suppl 360):52-53

Biziere K, Berger M (1990): Efficacy of a reversible monoamine oxidase-A inhibitor versus imipramine in subgroups of depressed patients. Acta Psychiatr Scand 82(Suppl 360):59-60

Bocksberger JP, Gachoud JP, Richard J, Dick P (1993): Comparison of the efficacy of moclobemide and fluvoxamine in elderly patients with a severe depressive episode. Eur Psychiatry 8:319-324

Botte L, Gilles C, Evrard JL, Mesters P, Wolfrum C, Berger M, Hellstern K, Ward J (1990): Moclobemide versus placebo in the treatment of depression: a multicentre study in Belgium. Acta Psychiatr Scand 82(Suppl 360): 42

Bougerol T, Uchida C, Gachoud JP, Kohler M, Mikkelsen H (1992): Efficacy and tolerability of moclobemide compared with fluvoxamine in depressive disorder (DSMIII). A French/Swiss double-blind trial. Psychopharmacol 106(Suppl):S102-S108

Casacchia M, Carolei A, Barba C, Frontoni M, Rossi A, Meco G, Zylberman MR (1984): A placebo-controlled study of the antidepressant activity of moclobemide, a new MAO-A Inhibitor. Pharmacopsychiatry 17:122-125

Casacchia M, Moll E (1990): Moclobemide (Ro 11-1163) versus imipramine in the treatment of depression. Acta Psychiatr Scand 82(Suppl 360):43

Casacchia M, Rossi A (1989): A comparison of moclobemide and imipramine in treatment of depression. Pharmacopsychiatry 22:152-155

Celada P, Perez J, Alvarez E, Artigas F (1992): Monoamine oxidase inhibitors phenelzine and brofaromine increase plasma serotonin and decrease 5-hydroxyindoleacetic acid in patients with major depression: relationship to clinical improvement. J Clin Psychopharmacol 12:309-315

Chen DT, Ruch R (1993): Safety of moclobemide in clinical use. Clin Neuropharmacol 16(Suppl 2):S63-S68
Chouinard G, Saxena BM, Nair PV, Kutcher SP, Bakish D, Bradwejn J, Kennedy SH, Sharma V, Remick A, KukhaMohamad SA, Beauclair L, Snaith J, D'Souza J (1993): Brofaromine in depression: a Canadian multicenter placebo trial and a review of standard drug comparative studies. Clin Neuropharmacol 16(Suppl 2):S51-S54

Civeira J, Cervera S, Giner J, Allen SR, Hellstern K, Malanowski H, Wirz R, Klar K (1990): Moclobemide versus clomipramine in the treatment of depression: a multicentre trial in Spain. Acta Psychiatr Scand 82(Suppl 360):48-49

Classen W, Laux G (1990): Psychometric alternations in treatment with the MAO-A-inhibitor moclobemide. J Neural Transm (Suppl 32):185-188

Danish University Antidepressant Group (1993): Moclobemide: A reversible MAO-A inhibitor showing weaker antidepressant effect than clomipramine in a controlled multicenter study. J Affective Disord 28:105-116

Depression Guideline Panel (1993): Clinical practice guideline number 5. In Depression in Primary Care, Vol 2, Treatment of Major Depression. Rockville, MD, U.S. Department of Health and Human Services Agency for Health Care Policy and Research. AHCPR Publication No. 93-0551.

De Vanna M, Kummer J, Agnoli A, Gentili P, Lorizio A, Anand R (1990): Moclobemide compared with secondgeneration antidepressants in elderly people. Acta Psychiatr Scand 82(Suppl 360):64-66

Dierick M, Cattiez P, Franck G, Burton P, Defleur J, Hermans W, Roelandts A, Wolfrum C, Berger M, Hellstern K, Ward J (1990): Moclobemide versus clomipramine in the treatment of depression: a double-blind multicentre study in Belgium. Acta Psychiatr Scand 82(Suppl 360):50-51

Eddy DM, Hasselblad V, Schacter R (1990): A Bayesian method for synthesizing evidence: The confidence profile method. Int J Technol Assess Health Care 6:31-55

Evans L, George T, O'Sullivan B, Mitchell P, Johnson G, Adena M (1992): An Australian multicentre study of moclobemide versus amitriptyline in the treatment of depression. Aust N Z J Psychiatry 26(3):454-458

Funke HJ, Moritz E, Hellstern K, Malanowski H (1990): Moclobemide versus clomipramine in the treatment of depression: a single-centre study. Acta Psychiatr Scand 82(Suppl 360):46-47

Gabelic L, Kuhn B (1990): Moclobemide (R011-1163) versus tranylcypromine in the treatment of endogenous depression. Acta Psychiatr Scand 82(Suppl 360):63

Gabelic I, Moll E (1990): Moclobemide (Ro 11-1163) versus desipramine in the treatment of endogenous depression. Acta Psychiatr Scand 82(Suppl 360):44-45

Gachoud JP, Dick P, Kohler M (1994): Comparison of the efficacy and tolerability of moclobemide and maprotiline in depressed patients treated by general practitioners. Clin Neuropharmacol 17(suppl 1):S29-S37

Gagiano CA, Hart GAD, Bodemer W, Schall R (1994): Moclobemide twice daily in the treatment of major depressive episode: a double-blind, multicenter comparison with different three-times-daily dosage schedules. Clin Neuropharmacol 17(Suppl 1):S1-S8

Gattaz WF, Vogel P, Kick H, Kohnen R (1995): Moclobemide 
versus fluoxetine in the treatment of inpatients with major depression. J Clin Psychopharmacol 15(Suppl 2):35S-39S

Geertz S, Bruynooghe F, De Cuyper H, Demeulemeester F, Haazen L (1995): Moclobemide versus fluoxetine for major depressive episodes. Clin Neuropharmacol 17(Suppl 1): S50-S57

Guelfi JD, Payan C, Fermanian J, Pedarriosse AM, Manfredi R (1992): Moclobemide versus clomipramine in endogenous depression. A double-blind randomized clinical trial. Br J Psychiatry 160:519-524

Guy W (1976): ECDEU Assessment Manual for Psychopharmacology. Washington DC, U.S. Government Printing Office. U.S. Department of Health, Education, and Welfare Publication No. 76-338

Haefely W, Burkard WP, Cesura A, Colzi A, Kettler R, Lorez HP, Martin JR, Moreau JL, Richards JG, Schaffner R, Scherschlicht R, Sepinwall J, Da Prada M (1993): Pharmacology of moclobemide. Clin Neuropharmacol 16 (suppl 2):S8-S18

Haefely W, Burkard WP, Cesura AM, Kettler R, Lorez HP, Martin JR, Richards JG, Scherschlicht R, De Prada M (1992): Biochemistry and pharmacology of moclobemide, a prototype RIMA. Psychopharmacol 196:S6-S14

Hamilton M (1960): A rating scale for depression. J Neurol Neurosurg Psychiatry 23: 56-62

Heinze G, Rossel L, Gabelic I, Galeano-Munoz J, Stabl M, Allen SR (1993): Double-blind comparison of moclobemide and tranylcypromine in depression. Pharmacopsychiat 26:240-245

Himmelhoch JM, Thase ME, Mallinger AG, Houck P (1991): Tranylcypromine versus imipramine in anergic bipolar depression. Am J Psychiatry 148:910-916

Hoencamp E, Haffmans PMJ, Dijken WA, Hoogduin CAL, Nolen WA, van Dyck R (1994): Brofaromine versus lithium addition to maprotiline. A double-blind study in maprotiline refractory depressed outpatients. J Affective Disord 30:219-227

Jauch R, Griesser E, Oesterhelt G, Arnold W, Meister W, Ziegler WH, Guentert TW (1990): Biotransformations of moclobemide in humans. Acta Psychiatr Scand 80(Suppl 360):87-90

Joffe RT, Bakish D (1994): Combined SSRI-moclobemide treatment of psychiatric illness. J Clin Psychiatry 55(1): 24-25

Johnston P (1968): Some observations upon a new inhibitor of monoamine oxidase in brain tissue. Biochem Pharmacol 17:1285-1297

Koczkas C, Holm P, Karlsson A, Nagy A, Ose E, Petursson H, Ulveras L, Wenedikter O (1989): Moclobemide and clomipramine in endogenous depression. A randomized clinical trial. Acta Psychiatr Scand 79:523-529

Korn A, Da Prada M, Raffesberg M, Allen S, Casic S (1988): Tyramine pressor effect in man: studies with moclobemide, a novel reversible monoamine oxidase inhibitor. J Neural Transmission Suppl 26:57-72

Kragh-Sorensen P, Muller B, Andersen JV, Buch D, Stage KB (1995): Moclobemide versus clomipramine in depressed patients in general practice. A randomized, doubleblind, parallel, multicenter study. J Clin Psychopharmacol 15(Suppl 2):24S-30S
Kusalic M, Engelsmann F, Bradwejn J (1993): Thyroid functioning during treatment for depression. J Psychiatry Neurosci 18:260-263

Larsen JK, Gjerris A, Holm P, Andersen J, Bille A, Christensen EM, Hoyer E, Jensen $H$, Mejhede A, Langagergaard A, Laursen AL, Nilakantan B, Olafsson K, Severin B, Rafaelsen OJ (1991): Moclobemide in depression: a randomized, multicentre trial against isocarboxazide and clomipramine emphasizing atypical depression. Acta Psychiatr Scand 84:564-570

Larsen J, Holm P, Hoyer E, Mejlhede A, Mikkelsen PL, Olesen A, Schaumburg E (1989): Moclobemide and clomipramine in reactive depression. A placebo-controlled randomized clinical trial. Acta Psychiatr Scand 79:530536

Larsen JK, Holm P, Mikkelsen PL (1984): Moclobemide and clomipramine in the treatment of depression. A randomized clinical trial. Acta Psychiatr Scand 70:254-260

Laux G, Volz HP, Möller HJ (1995): Newer and older monoamine oxidase inhibitors. A comparative profile. CNS Drugs 3(Suppl 2):145-158

Lavori P (1992): Clinical trials in psychiatry: Should protocol deviation censor patient data? Neuropsychopharmacol 6(1):39-63

Lavian G, Finberg PM, Youdim MBH (1993): The advent of a new generation of monoamine oxidase inhibitor antidepressants: pharmacologic studies with moclobemide and brofaromine. Clin Neuropharmacol 16(Suppl 2):S1S7

Lecrubier Y, Guelfi JD (1990): Efficacy of reversible inhibitors of monoamine oxidase-A in various forms of depression. Acta Psychiatr Scand 82(Suppl 360):18-23

Lemoine P, Mirabaud C (1992): A double-blind comparison of moclobemide and toloxatone in out-patients presenting a major depressive disorder. Psychopharmacol 106(Suppl):S118-S119

Lingjaerde O, Reichborn-Kjennerud T, Haggag A, Gartner I, Narud K, Berg EM (1993): Treatment of winter depression in Norway. II. A comparison of the selective monoamine oxidase A inhibitor moclobemide and placebo. Acta Psychiatr Scand 88:372-380

Lingjaerde $\mathrm{O}$, Jorgensen J, Storen R, Thomle S, Wendt Raeder L, Ruud LE, Schetelig E, Sveaas HK, Leivestad O (1995): A double-blind comparison of moclobemide and doxepin in depressed general practice patients. Acta Psychiatr Scand 92:125-131

Lipper S, Murphy DL, Slater S, Buchsbaum MS (1979): Comparative behavioral effects of clorgyline and pargyline in man: a preliminary evaluation. Psychopharmacol 62:123-128

Lonnqvist J, Sihvo S, Syvalahti E, Kiviruusu O (1994b): Moclobemide and fluoxetine in atypical depression: a double-blind trial. J Affective Disorders 32(3):169-177

Lonnqvist J, Sihvo S, Syvalahti E, Sintonen H, Kiviruusu O, Pitkanen H (1995): Moclobemide and fluoxetine in the prevention of relapses following acute treatment of depression. Acta Psychiatr Scand 91(3):189-194

Lonnqvist J, Sintonen H, Syvalahti E, Appelberg B, Koskinen T, Manniko T, Mehtonen O-P, Naarala M, Sihvo S, Auvinen J, Pitkanen H (1994a): Antidepressant efficacy 
and quality of life in depression: a double-blind study with moclobemide and fluoxetine. Acta Psychiatr Scand 89(6):363-369

Macher JP, Mirabaud C (1992): A double-blind comparison of moclobemide and amineptine in the treatment of depressed out-patients. Psychopharmacol 106(Suppl): S116-S117

Mann JJ, Aarons SF, Wilner PJ, Keilp JG, Sweeney JA, Pearlstein T, Frances AJ, Kocsis JH, Brown RP (1989): A controlled study of the antidepressant efficacy and side effects of (-)-Deprenyl. A selective monoamine oxidase inhibitor. Arch Gen Psychiatry 46:45-50

Moll E, Hetzel W (1990): Moclobemide (R0 11-1163) safety in depressed patients. Acta Psychiatr Scand 82(Suppl 360):69-70

Möller HJ, Volz HP (1992): Brofaromine in major depressed patients: a controlled clinical trial versus imipramine and open follow-up of up to one year. J Affective Disord 26:163-172

Möller HJ, Volz HP (1993): Brofaromine in elderly major depressed patients-a comparative trial versus imipramine. Eur Neuropsychopharmacol 3:501-510

Möller JH, Wendt G (1989): Brofaromin-ein selektiver, reversibler and kurzwirksamer MAOI-A Hemmer. Psychiatr Prax 16:32-36

Nair NPV, Ahmed SK, Ng Ying Kin NMK, West TEG (1995a): Reversible and selective inhibitors of monoamine oxidase $\mathrm{A}$ in the treatment of depressed elderly patients. Acta Psychiatr Scand 91(Suppl 386):28-35

Nair NPV, Amin M, Holm P, Katona C, Klitgaard N, Ng Ying Kin NMK, Kragh-Sorensen P, Kuhn H, Leek CA, Stage KB (1995b): Moclobemide and nortriptyline in elderly depressed patients. A randomized, multicentre trial against placebo. J Affective Disord 33:1-9

Newburn GM, Fraser AR, Menkes DB, Mullen PE (1990): A double-blind trial of moclobemide versus amitriptyline in the treatment of depressive disorders. Aust N Z J Psychiatry 24:475-479

Nolen WA, Haffmans PMJ, Bouvy PF, Duivenvoorden HJ (1993): Monoamine oxidase inhibitors in resistant major depression. A double-blind comparison of brofaromine and tranylcypromine in patients resistant to tricyclic antidepressants. J Affective Disord 28:189-197

Nolen WA, Hoencamp E, Bouvy PF, Haffmans PMJ (1993): Reversible monoamine oxidase-A inhibitors in resistant major depression. Clin Neuropharmacol 16(Suppl 2):S69 S76

Norman TR, Ames D, Burrows GD, Davies B (1985): A controlled study of a specific MAO A reversible inhibitor (R0 11-1163) and amitriptyline in depressive illness. J Affective Disord 8:29- 35

Nutt D, Glue P (1989): Monoamine oxidase inhibitors: rehabilitation from recent research? Br J Psychiatry 154:287-291

Ose E, Holm P (1992): Moclobemide and placebo in mild major depression: a double-blind randomized trial. Psychopharmacol 106(Suppl):S114-S115

Pancheri P, Delle Chiaie R, Donnini M, Seripa S, Gambino C, Vicario E, Trillo L (1994): Effects of moclobemide on depressive symptoms and cognitive performance in a geriatric population: a controlled comparative study versus imipramine. Clin Neuropharmacol 17(Suppl 1):S58-S73

Paykel ES (1995): Clinical efficacy of reversible and selective inhibitors of monoamine oxidase A in major depression. Acta Psychiatr Scand 91(suppl 386):22-27

Philipp M, Kohnen R, Benkert O (1993): A comparison study of moclobemide and doxepin in major depression with special reference to effects on sexual dysfunction. Int Clin Psychcopharmacol 7:149-153

Potter WZ, Murphy DL, Wehr TA, Linnoila M, Goodwin FK (1982): Clorgyline. A new treatment for patients with refractory rapid-cycling disorder. Arch Gen Psychiatry 39:505-510

Prien RF, Carpenter LL, Kupfer DJ (1991): The definition and operational criteria for treatment outcome of major depressive disorder: a review of the current research literature. Arch Gen Psychiatry 48:796-800

Quitkin FM, Stewart JW, McGrath PJ, Nunes E, OcepekWelikson K, Tricamo E, Rabkin JG, Klein DF (1993): Further evidence that a placebo response to antidepressants can be identified. Am J Psychiatry 150:566-570

Reynaert C, Parent M, Mirel J, Janne P, Haazen L (1995): Moclobemide versus fluoxetine for a major depressive episode. Psychopharmacol 118:183-187

Rimon R, Jaaskelainen J, Kaatinen P, Kalli A, Kilponen E, Koskinen T, Nikkila H, Pirttipera V, Seppala J (1993): Moclobemide versus imipramine in depressed outpatients: a double-blind multi-centre study. Int Clin Psychopharmacol 7:141-147

Robinson DS, Lerfald SC, Bennett B, Laux D, Devereaux E, Kayser E, Kayser A, Corcella J, Albright D (1991): Continuation and maintenance treatment of major depression with the monoamine oxidase inhibitor phenelzine: a double-blind, placebo-controlled discontinuation study. Psychopharmacol Bull 27:31-39

Robinson DS, Nies A, Ravaris CL, Ives JO, Bartless D (1978): Clinical pharmacology of phenelzine. Arch Gen Psychiatry 35:629-635

Rossel L, Moll E (1990): Moclobemide versus tranylcypromine in the treatment of depression. Acta Psychiar Scand 82(Suppl 360):61-62

Schiwy W, Heath WR, Delini-Stula A (1989): Therapeutic and side-effect profile of a selective and reversible MAOA inhibitor, brofaromine. Results of dose-finding trials in depressed patients. J Neural Transm 28(Suppl):33-44

Stabl M, Kasas A, Blajev B, Bajetta G, Zochling R, HolsboerTrachsler E, Realini R, Schaublin-Loidl M (1995): A double-blind comparison of moclobemide and thioridazine versus moclobemide and placebo in the treatment of refractory, severe depression. J Clin Psychopharmacol 15(Suppl 2):41S-45S

Stefanis CN, Alevizos B, Papadimitrou GN (1984): Controlled clinical study of moclobemide (R0 11-1163), a new MAO inhibitor, and desipramine in depressive patients. In Tipton KF, Dostert P, Strolin-Benedetti M (eds), Monoamine Oxidase and Disease. Prospects for Therapy with Reversible Inhibitors. London, Academic Press, pp 377-392

Stefanis CN, Merz-Frei K (1990). Moclobemide (R0 11-1163) in long-term treatment. Acta Psychiatr Scand 82(Suppl 360):67-68 
Steinmeyer EM, Vorbach EU, Arnoldt KH (1993): Efficacy and safety of moclobemide compared with maprotiline in treatment of major depressive disorder-A doubleblind multicenter study with parallel groups. Pharmacopsychiatry 26:246-253

Stewart JW, Tricamo, E, McGrath PJ, Quitkin FM (1997): Prophylactic efficacy of phenelzine and imipramine in chronic atypical depression: likelihood of recurrence on discontinuation after 6 month's remission. Am J Psychiatry 154(1):31-36

Sunderland T, Cohen RM, Molchan S, Lawler BA, Mellow AM, Newhouse PA, Tariot PN, Mueller EA, Murphy DL (1994): High-dose selegiline in treatment-resistant older depressive patients. Arch Gen Psychiatry 51:607-615

Thase ME, Kupfer DJ (1987): Characteristics of treatmentresistant depression. In Zohar J, Belmaker RH (eds), Treating Resistant Depression. New York, PMA Publishing Co., pp 23-45

Thase ME, Frank E, Mallinger A, Hamer T, Kupfer DJ (1992b): Treatment of imipramine-resistant recurrent depression: III. Efficacy of monoamine oxidase inhibitors. J Clin Psychiatry 53:5-11

Thase ME, Mallinger AG, McKnight D, Himmelhoch JM (1992a): Treatment of imipramine resistant recurrent depression: IV. A double-blind, crossover study of tranylcypromine in anergic bipolar depression. Am J Psychiatry 159:195-198

Thase ME, Rush AJ (1995): Treatment resistant depression. In Bloom FE, Kupfer DJ (eds), Psychopharmacology: The Fourth Generation of Progress. New York, Raven Press, pp 1081-1097

Thase ME, Sullivan LR (1995): Relapse and recurrence of depression: a practical approach for prevention. CNS Drugs 4:261-277

Thase ME, Trivedi MH, Rush AJ (1995): MAOIs in the contemporary treatment of depression. Neuropsychopharmacol 12(3):185-219

Tiller JWG (1990): Antidepressants, alcohol and psychomotor performance. Acta Psychiatr Scand 82(Suppl 360): 13-17

Tiller J, Maguire K, Davies B (1990): A sequential doubleblind controlled study of moclobemide and mianserin in elderly depressed patients. Int J Geriatr Psychiatry 5:199-204

Tiller J, Schweitzer I, Maguire K, Davies B (1989): Is diazepam an antidepressant? Br J Psychiatry 155:483-489

Ucha Udabe R, Marquez CA, Traballi CA, Portes N (1990): Double-blind comparison of moclobemide, imipramine and placebo in depressive patients. Acta Psychiatr Scand 82(Suppl 360):54-56

UK Moclobemide Study Group (1994): A multicentre comparative trial of moclobemide, imipramine and placebo in major depressive disorder. Int Clin Psychopharmacol 9:109-113

Van Vliet IM, Westenberg HGM, Den Boer JA (1993): MAOI inhibitors in panic disorder. Clinical effects of treatment with brofaromine: a double blind placebo controlled study. Psychopharmacol 112:483-489

Vaz-Serra A, Figueira ML, Firmino H, Albuquerque AJ, Jara JM, Pestana LC (1994): Multicenter double-blind study of moclobemide and maprotiline. Clin Neuropharmacol 17(Suppl 1):S38-S49

Versiani M, Nardi D, Capponi R, Costa DA, Magistris H, Ucha Udabe R (1992): Moclobemide compared with imipramine in the treatment of chronic depression (dysthymia DSM-III-R): a double-blind placebo-controlled trial. Clin Neuropharmacol 15(Suppl 1):148b

Versiani M, Nardi AE, Figueira ILV, Stabl M (1990): Tolerability of moclobemide, a new reversible inhibitor of monoamine oxidase-A, compared with antidepressants and placebo. Acta Psychiatr Scand 82(Suppl 360):24-28

Versiani M, Nardi AE, Mundim FD, Alves A, Schmid-Burgk W (1990): Moclobemide, imipramine and placebo in the treatment of major depression. Acta Psychiatr Scand 82(Suppl 360):57-58

Versiani M, Oggero U, Alterwain P, Capponi R, Dajas F, Heinze-Martin G, Marquez CA, Polero MA, Rivero-Almanzor LE, Rossel L, Schmid-Burck W, Ucha Udabe R. (1989): A double-blind comparative trial of moclobemide v. imipramine and placebo in major depressive episodes. Br J Psychiatry 155(Suppl 6):72-77

Volz HP, Faltus F, Magyar I, Möller HJ (1994a): Brofaromine in treatment-resistant depressed patients-a comparative trial versus tranylcypromine. J Affective Disord 30:209-217

Volz HP, Gleiter CH, Struck M, Möller HJ (1995): Brofaromine. Insight into the nature of drug development. CNS Drugs 3:1-8

Volz HP, Heimann H, Bellaire J, Laux G, Möller HJ (1994b): Brofaromine in non-endogenous major depressed inpatients-Results of a preliminary dose-finding trial versus tranylcypromine. Pharmacopsychiatry 27:152-158

Volz H-P, Möller H-J (1994): Antidepressant drug therapy in the elderly-A critical review of the controlled clinical trials conducted since 1980. Pharmacopsychiat 27:93100

Waldmeier PC, Glatt A, Jaekel J, Bittiger H (1993): Brofaromine: A monoamine oxidase-A and serotonin uptake inhibitor. Clin Neuropharmacol 16(Suppl 2):S19-S24

Williams R, Edwards RA, Newburn GM, Mullen R, Menkes DB, Segkar C (1993): A double-blind comparison of moclobemide and fluoxetine in the treatment of depressive disorders. International Clin Psychopharmacol 7:155-158

Youdim MBH (1995): The advent of selective monoamine oxidase A inhibitor antidepressants devoid of the cheese reaction. Acta Psychiatr Scand 91(Suppl 386):5-7

Youdim MBH, Finberg JPM, Tipton KF (1988): Monoamine oxidase. In Trendelemburg U, Weiner U (eds), Catecholamine II. Berlin, Springer Verlag, pp 192-199 